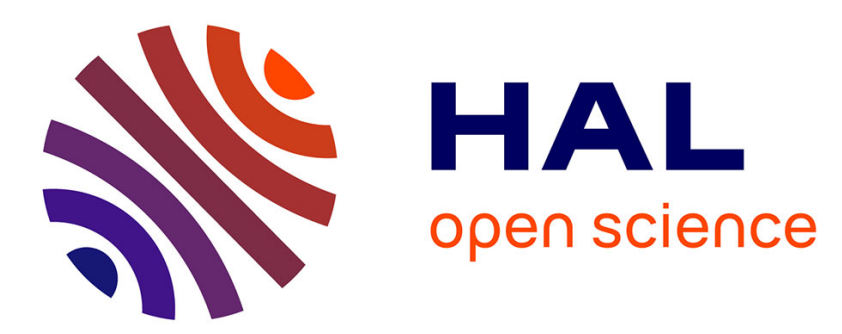

\title{
Millimeter-Wave Heating in In Vitro Studies: Effect of Convection in Continuous and Pulse-Modulated Regimes
}

Rosa Orlacchio, Maxim Zhadobov, Stanislav I. Alekseev, Denys Nikolayev, Ronan Sauleau, Yann Le Page, Yves Le Drean

\section{- To cite this version:}

Rosa Orlacchio, Maxim Zhadobov, Stanislav I. Alekseev, Denys Nikolayev, Ronan Sauleau, et al.. Millimeter-Wave Heating in In Vitro Studies: Effect of Convection in Continuous and Pulse-Modulated Regimes. Bioelectromagnetics, 2019, 40 (8), pp.553-568. 10.1002/bem.22223 . hal-02355635

\section{HAL Id: hal-02355635}

\section{https://hal-univ-rennes1.archives-ouvertes.fr/hal-02355635}

Submitted on 9 Nov 2020

HAL is a multi-disciplinary open access archive for the deposit and dissemination of scientific research documents, whether they are published or not. The documents may come from teaching and research institutions in France or abroad, or from public or private research centers.
L'archive ouverte pluridisciplinaire HAL, est destinée au dépôt et à la diffusion de documents scientifiques de niveau recherche, publiés ou non, émanant des établissements d'enseignement et de recherche français ou étrangers, des laboratoires publics ou privés. 


\title{
Millimeter-wave heating in in vitro studies: effect of convection in continuous and pulse- modulated regimes
}

\author{
Rosa Orlacchio ${ }^{1,2}$, Maxim Zhadobov ${ }^{1}$, Stanislav I. Alekseev ${ }^{3}$, Denys Nikolayev ${ }^{4}$, \\ Ronan Sauleau ${ }^{1}$, Yann Le Page ${ }^{5}$, and Yves Le Dréan ${ }^{5}$ \\ ${ }^{1}$ Univ Rennes, CNRS, IETR (Institut d'Electronique et de Télécommunication de Rennes) - \\ UMR 6164, F-35000 Rennes, France \\ ${ }^{2}$ Univ. Limoges, CNRS, XLIM, UMR 7252, F-87000 Limoges, France \\ ${ }^{3}$ Institute of Cell Biophysics of Russian Academy of Sciences, Pushchino, Moscow Region, \\ Russia \\ ${ }^{4}$ Microwaves and Antennas Group (MAG), EPFL, Lausanne, Switzerland \\ ${ }^{5}$ Univ Rennes, Inserm, EHESP, IRSET (Institut de Recherche en Santé, Environnement et \\ Travail) UMR_S 1085, F-35000 Rennes, France
}

\author{
Corresponding author \\ Rosa Orlacchio \\ Institute of Electronics and Telecommunications of Rennes (IETR) \\ University of Rennes 1 \\ 11D, 263 av. du G. Leclerc, 35042 Rennes, France \\ Email: rosa.orlacchio@hotmail.it
}

\section{Running title \\ Convection in in vitro experiments at MMW frequencies}

Grant sponsors: French Agency for Food, Environmental and Occupational Health and Safety (ANSES) through NEAR 5G and MEMO projects; the EU through the European Regional Development Fund (ERDF), Brittany Region, Ministry of Higher Education and Research, Rennes Métropole and Conseil Départemental, through the CPER Project SOPHIE / STIC \& Ondes; the French National Center for Scientific Research (CNRS) through INSIS grant "Bioelectromagnetics: from engineering to biomedical applications"

Conflict of interest: none 


\begin{abstract}
Shallow penetration of millimeter waves (MMW) and non-uniform illumination in in vitro experiments result in a non-uniform distribution of the specific absorption rate (SAR). These SAR gradients trigger convective currents in liquids affecting transient and steady-state temperature distributions. We analyzed the effect of convection on temperature dynamics during MMW exposure in continuous-wave (CW) and pulsed-wave (PW) amplitudemodulated regimes using micro-thermocouples. Temperature rise kinetics are characterized by the occurrence of a temperature peak that shifts to shorter times as the SAR of the MMW exposure increases and precedes initiation of convection in bulk. Furthermore, we demonstrate that the liquid volume impacts convection. Increasing the volume results in earlier triggering of convection and in a greater cooling rate after the end of exposure. In PW regimes, convection strongly depends on the pulse duration that affects the heat pulse amplitude and cooling rate. The latter results in a change of the average temperature in PW regime.
\end{abstract}

\title{
Keywords
}

millimeter waves; thermal dosimetry; local heating; in vitro experiments; Grashof number 


\section{Introduction}

Therapeutic applications of heat date back to the writing of Hippocrates [Habash et al., 2006]. Modern thermal therapy emerged in the mid-1960s [Hahn, 1974], and now its main application is in oncology [Mellala et al., 2017]. At the cellular level, heating induces a complex cascade of phenomena, which may lead to cell death through different pathways. Cytotoxicity and radiosensitization are the main cellular responses to heat shock [Roti Roti, 2008], which are exploited in thermal ablation and hyperthermia, respectively.

Different power sources have been used to induce heating in biological tissues, including ultrasound and various bands of the electromagnetic spectrum, i.e., radiofrequency (RF), microwave (MW), ultraviolet (photothermal therapy), and light (lasers) [Melalla et al., 2017]. In the MW range, devices used for thermal ablation generally operate in the Industrial Scientific Medical (ISM) bands around $434 \mathrm{MHz}, 915 \mathrm{MHz}$, and $2.45 \mathrm{GHz}$. However, numerous advantages of higher frequencies, such as higher spatial resolution or compact size of radiating structures, have motivated exploration of $\mathrm{MW}$ up to $18 \mathrm{GHz}$ for local tumor ablation (9.2 GHz [Hodgson et al., 1999], $10 \mathrm{GHz}$ [Luyen et al., 2014], $14.5 \mathrm{GHz}$ [Hancock et al., 2013], and $18 \mathrm{GHz}$ [Yoon et al., 2011]). Furthermore, it has been demonstrated that the lower part of the millimeter-wave (MMW) range (i.e., 20-100 GHz) can be exploited to selectively focus heating within the skin layers as an alternative approach for the non-invasive treatment of skin cancers, such as melanoma [Zhadobov et al., 2015].

In general, during thermal therapies, heat is delivered in a continuous manner. However, pulsed electromagnetically induced heating could induce stronger responses in cells [Bedoya et al., 2014]. Short thermal pulses may provide several advantages [Hunt et al., 1991; Kim et al., 1998]: i) to decrease treatment duration reducing patient discomfort and overall costs, ii) to eliminate or reduce the influence of blood perfusion, iii) to eliminate or reduce thermotolerance. 
In vitro experiments are performed to quantify the thermal injury at the cellular and molecular level and, in particular, to determine kinetic models of heat-induced damage [He and Bischof, 2003]. At MMW frequencies, temperature measurements at the cell monolayer level are extremely challenging due to very local power absorption. A methodology to measure the temperature rise during in vitro exposure in the MMW band was reported in Zhadobov et al. [2017]. It was shown that a micro-thermocouple (TC) could be used to measure the local continuous-wave (CW) and pulsed-wave (PW) MMW-induced heating. The MMW and MW power density distributions in in vitro experiments are not uniform [Guy et al., 1999; Zhao, 2005; Zhadobov et al., 2008, 2012b; Boriskin et al., 2013; Paffi et al., 2015]. This results in the generation of temperature gradients that lead to the formation of density differences in the liquid. The latter directs the natural free convection flow from the bottom of the well to the top when the tissue culture plate is exposed from the bottom. The surrounding cooler liquid moves to the bottom and after heating reiterates the convection flow [Ayappa et al., 1994; Tanasawa, 1995; Zhang et al., 2000; Incropera et al., 2007].

Natural convection has been widely investigated and well discussed in the literature [Bird et al., 1960; Chapman, 1967; Lienhard and Lienhard, 1981; Sekins and Emery, 1982; Cengel, 2004; Bejan, 2013]. Since the solution of the differential equations describing convective heat transfer is complicated, dimensionless groups such as Rayleigh (Ra) and Grashof (Gr) numbers are used to characterize the natural convection flow. Ra number defines the threshold separating the quiescent from convective regimes. For Ra numbers below some critical value, the fluid is stable. When the Ra number is above the critical value, the onset of natural convection occurs. For convection of fluid between solid boundaries, the critical Ra number is calculated to be 1708.8 [Jeffreys, 1926]. For other combinations of boundaries, the critical Ra number is $<1708.8$ [Heitz and Westwater, 1971]. The Grashof number is defined by the ratio of the buoyant forces on the fluid to the viscous forces. It is 
used to define the convection type, i.e., laminar or turbulent. For Gr numbers exceeding $10^{9}$, the convective flow is considered to be turbulent [Sekins and Emery, 1982].

The initiation of convection in liquids by MMW and MW exposures has been described in several studies [Sharov et al., 1983; Khizhnyak and Ziskin, 1996; Betskii et al., 2000; Cueille et al., 2008; Paffi et al., 2015; Zhadobov et al., 2017]. Convection during in vitro bioelectromagnetic experiments affects the temperature dynamics and distribution [Sandblom and Theander, 1991; Pickard et al., 1999], causing the formation of a toroidal vortex [Khizhnyak and Ziskin, 1996] and temperature drop in the irradiated spot [Wessapan and Rattanachecho, 2014; Zhadobov et al., 2017]. Additionally, liquid motion caused by convective currents may perturb the local concentration of oxygen and nutrients near the surface of the cells [Paffi et al., 2015]. Thus convection-induced thermodynamic events, absent in non-exposed sham samples, may lead to misinterpretation of the biological outcome.

Therefore, accurate thermal dosimetry in in vitro studies requires a detailed analysis of convection arising in the heated liquid. The aim of this study was to analyze in detail local convection in liquid samples exposed to MMW in CW and PW regimes. Using several models, including culture medium, distilled water, and agar gel (convection-free waterequivalent model), we investigated the effects of convection on heating by comparing localized temperature kinetics recorded in liquids to that in water-equivalent gel without convection. Convection was studied as a function of several parameters, which may differ from one in vitro experiment to another, including i) viscosity, ii) specific absorption rate (SAR), iii) liquid volume, and iv) duration of a thermal pulse.

\section{Materials and methods}

\section{Exposure system}


The exposure system consisted of two main units: i) an exposure chamber, and ii) a signal generator unit (Fig. 1).

Exposure chamber. All exposures were carried out inside a MEMMERT UNE 400 incubator (Memmert, Schwabach, Germany) set at $32^{\circ} \mathrm{C}$. Samples under test (SUTs), described in the following section, were placed into one well of a 12-well tissue culture plate (TCP) (353072, Microtest 96, Becton Dickinson, Franklin Lakes, NJ) made of polystyrene. Each well was $22.09 \mathrm{~mm}$ in diameter. Exposures were performed from the bottom by an open-ended rectangular waveguide (WG) WR15 (aperture size $3.81 \times 1.905 \mathrm{~mm}^{2}$ ) located $5 \mathrm{~mm}$ from the bottom of TCP. The latter was set on a $5 \mathrm{~mm}$-thick plastic support with a 3.5 $\mathrm{cm}$ hole in diameter, centered with the exposed well. The open-ended WG was connected to an MMW generator (QuinStar Technology, Torrance, CA) by means of standard WR15 waveguides (their total length is $27.5 \mathrm{~cm}$ ).

Signal generator unit. A high-power generator (QuinStar Technology, Torrance, CA) operating at $58.4 \mathrm{GHz}$ was used as a source. In the PW regime, a programmable power supply HMP (Hameg Instruments, Hampshire, UK) provided control voltage, enabling amplitude modulation of MMW to create thermal pulses with duration ranging from $1.0 \mathrm{~s}$ to $6.5 \mathrm{~s}$.

The input power of the open-ended WG was measured before experiments using a V-band Agilent V8486A power meter (Agilent Technologies, Santa Clara, CA). The measurements were performed by replacing the open-ended WG by the power meter sensor. To this end, the open-ended WG was disconnected from the feeding WG, and the power meter sensor was directly connected to the feeding WG.

\section{Sample under test}

The heating dynamics were studied in four samples detailed below. 
- SUT 1. Distilled water.

- SUT 2. Culture medium. Dulbecco's modified eagle medium (DMEM) was supplemented with $8 \%$ of fetal calf serum, $1 \%$ antibiotics, and $1 \%$ L-glutamine made of amino acids, vitamins, inorganic salts, and other components (D-Glucose, Phenol Red, Sodium Pyruvate). At MMW frequencies, the electromagnetic properties of the culture medium are close to those of pure water, as discussed in detail in the sub-section "Heating in different media."

- SUT 3. Melanoma cell monolayer was attached to the bottom of the exposed well and covered by the culture medium. A375 melanoma cells [Giard et al., 1973] were seeded at a concentration of $10^{5}$ cells in $2 \mathrm{ml}$ of the same culture medium as in SUT 2. Before exposure, the medium was replaced by DMEM without sodium bicarbonate (Ref. 12800-017, Thermo Fisher Scientific, Waltham, MA) containing 4.6 mM of Hepes (Thermo Fisher Scientific) to maintain constant $\mathrm{pH}$ in the non-gassed incubator of the exposure system [Le Quément et al., 2014].

- SUT 4. Agar gel (98.5\% of distilled water and $1.5 \%$ of agar) was used as a reference water-equivalent model without convection. Except for viscosity, both electromagnetic [Chahat et al., 2012] and thermophysical parameters [Ergodgu, 2008] of the agar phantom closely matched those of pure water. The fabrication procedure of the agar phantom is detailed in Chahat et al. [2012] and outlined hereafter. Distilled water was heated up to boiling. Then agar was progressively added and stirred. The obtained mixture was poured into a well of a 12-well TCP where a thin TC was attached to the bottom. Afterwards, the sample was left at room temperature to solidify.

The electromagnetic properties of the SUTs at $58.4 \mathrm{GHz}$ are given in Table 1. The complex permittivity of the culture medium, water, and agar gel was measured at room temperature using an open-ended coaxial slim probe (Agilent Technologies) [Zhadobov et al., 
2012a]. Note that according to the free water temperature-dependent model [Ellison, 2007], a variation of electromagnetic properties in the $22-52{ }^{\circ} \mathrm{C}$ range (room temperature and maximum temperature reached during the experiments, respectively) resulted in SAR variation of less than $2 \%$. In simulations, the permittivity and conductivity were considered to be temperature-independent, taking into account this small variation of the SAR and the fact that the permittivity measurements at temperatures exceeding the room temperature resulted in increased uncertainty. Complex permittivity of polystyrene was determined using a freespace technique with a transmission/reflection quasi-optical setup and ABmm millimeterwave vector network analyzer [Guraliuc et al., 2014].

Thermal and mechanical properties of water, culture medium, and agar gel are provided in Table 2 . Heat capacity $C$ and thermal conductivity $k$ of water and culture medium were measured with a differential scanning calorimeter (DSC, Q200, TA Instruments, New Castle, DE) and a thermal conductivity analyzer (Tci, C-THERM Technologies, New Brunswick, Canada), respectively, within the $32-52{ }^{\circ} \mathrm{C}$ range (minimum and maximum temperatures in our experiments). In this range, the heat capacity variation was less than $1 \%$, and the thermal conductivity increased by $2 \%$. The heat capacity and thermal conductivity values of agar gel are well known; they are close to those of water as shown in several studies [Goodhew and Griffiths, 2004; Erdogdu, 2008; Sun, 2007; Zhang et al., 2011]. The density $\rho$ of water, culture medium, and agar gel were taken from Incropera et al. [2007], Paffi et al. [2015], and Erdogdu [2008], respectively.

The viscosity $\eta$ of the liquid samples plays an important role in convection initiation, which occurs as a result of the fluid motion due to density changes arising from heating [Zhang et al., 2011]. The viscosity of water has been characterized in several studies [Incropera et al., 2007; IAPWS 2008]. The viscosity of culture medium is $7 \%$ higher than that of water [Fröhlich et al., 2013]. The viscosity of the agar gel is much higher than liquids. In 
the $32-52{ }^{\circ} \mathrm{C}$ interval, agar gel can be considered as solid and its viscosity diverges towards infinity [Fernández et al., 2008].

\section{Electromagnetic dosimetry}

The finite integration technique (FIT) solver of CST Microwave Studio 2018 (CST Computer Simulation Technology, Dassault Systemes, Darmstadt, Germany) was used for numerical analysis. The exposure scenario (Fig. 2) is represented by one well of the TCP and feed antenna to reduce the computational volume represented in each simulation to about 45 million mesh cells. This assumption is justified by the fact that during MMW exposure, the absorbed energy is mainly localized at the bottom of the plate close to the well axis, making the contribution of reflections from the neighboring empty wells negligible.

Non-uniform adaptive meshing was used to carefully account for high SAR gradients at the bottom of the exposed liquid. The simulations were performed using a mesh with a cell size ranging from $2 \mu \mathrm{m}$ in liquid up to $340 \mu \mathrm{m}$ in free space. Perfectly matched layers absorbing boundary conditions were used. Minimal distance between the culture plate and boundaries was set to $\lambda_{0} / 4$.

\section{Temperature measurements}

Among the methods used for thermal dosimetry at MMW frequencies (infrared thermometry [Chahat et al., 2012], thermo-sensitive liquid crystals [Kojima et al., 2015], and fiber optic thermometry [Zhadobov et al., 2012b]), microscale TC allows for accurate measurement of the local temperature with a time constant of the order of $\mu \mathrm{s}$. This technique is particularly well-adapted to measure the sharp MMW-induced thermal pulses [Zhadobov at al., 2017]. 
In this study, local temperature at the bottom of the well was measured using a $\mathrm{K}$ type TC (RS Components, Corby, UK) probe with a lead diameter of $75 \mu \mathrm{m}$. To record the temperature, we used Thermocouple Reference design (Microchip Technology, Chandler, AZ) with a sampling interval of $156 \mathrm{~ms}$. The tip of the TC was aligned with the exposure beam axis, which coincided with the axis of the exposed well. The leads of the TC were located close to each other and laid on the bottom of the well, perpendicular to the E plane ( $x z$ plane, Fig. 2). Such an orientation of the TC prevents possible artifacts in temperature measurements related to induced currents [Cetas, 1982; Dunscombe et al., 1986, 1988; Constable et al., 1987; Alekseev and Ziskin, 2001, 2003; Alekseev et al., 2011]. This was verified experimentally by performing measurements in an empty well with and without exposure. Reproducibility was assured by repeating measurements at least four times per condition if not stated otherwise.

\section{Results}

\section{Power absorption in SUT}

\section{a. Computed SAR}

Table 3 summarizes the peak values of SAR computed for WG input power of $195 \mathrm{~mW}$ (the same power level as the one used to determine SAR based on temperature measurements [see the next sub-section]). From the electromagnetic point of view, the differences between the four SUTs are almost negligible (maximum deviation of SAR with respect to that in water is within $2 \%$ ). SAR rapidly decreases along all the directions. For instance, the SAR drop from $50 \%$ to $10 \%$ levels corresponds to $(x=3.8, y=2.5$, or $z=0.1) \mathrm{mm}$ and $(x=8.7, y=5.3$, or $z=0.37) \mathrm{mm}$, respectively (dotted lines in Fig. 3). 
Note that introducing the TC results in a local increase of SAR close to the TC tip (in E plane) by about $5 \%$ (Fig. 3). This is mainly attributed to diffraction around the TC. However, due to heat conduction, this local peak of SAR has a very limited impact on the heating and therefore practically does not affect temperature measurements. Our simulations performed using CST estimated the difference to be less than $1 \%$, confirming the results of previous studies. Indeed, as shown by Alekseev and Ziskin [2001] and Alekseev et al. [2011], small thermocouples, with a diameter $\leq 0.1 \mathrm{~mm}$, provide accuracy of the temperature rise rate measurements in samples exposed to MMW within 3\%.

The effect of a cell monolayer was numerically investigated in detail at 42 and $58.4 \mathrm{GHz}$ for identical exposure conditions in Zhadobov et al. [2017] and Alekseev et al. [2017]. They showed that the presence of a $10-\mu$ m-thick monolayer resulted in a $1 \%$ reduction of the total power absorbed in the sample and only slightly impacted the SAR distribution in the culture medium. These results were obtained for tightly packed cells, and in experiments, typical density of cells is even smaller. Therefore, the effect of cell monolayer on power absorption is insignificant.

\section{b. Temperature-based determination of SAR}

To experimentally validate the numerical results, the following temperature-based approach was used to determine SAR [Zhadobov et al., 2017].

The thermal noise obscuring the initial phase of heating kinetics significantly reduces the accuracy of the direct measurement of the initial temperature rise rate. As shown in Alekseev and Ziskin [2003], the most reliable way of determining SAR based on temperature measurements is to fit a thermal model to experimental heating kinetics, and then calculate the initial temperature rise rate from the model. Temperature rise rate at the initial phase of exposure is proportional to the SAR [Alekseev and Ziskin, 2003]. 
Temperature kinetics were fitted to the thermal model described in Zhadobov et al. [2017] for the first 10-15 s of exposure at $195 \mathrm{~mW}$ (Fig. 4). The fitting curves (black solid lines) closely match the experimental data. The measured peak SAR values are given in Table 3 as mean $\pm \mathrm{SD}$ determined from five independent recordings. The SAR differences between water, culture medium, and agar gel are within $3 \%$, confirming the numerical results (Table $3)$.

The SARs determined from the temperature measurements are below the computed values (e.g., $19 \%$ less for water). This difference results from the $20 \%$ underestimation of the temperature measurement with $75-\mu \mathrm{m}$ TC as demonstrated in Zhadobov et al. [2017] for identical exposure conditions. Corrected results accounting for this underestimation (corrected $\mathrm{SAR}_{\text {peak }}$ in Table 3) are in a good agreement with computations. Under different experimental conditions, additional analysis of the accuracy of TC measurements may be required.

\section{CW induced heating}

\section{a. Heating in different media}

Figure 5a shows the temperature dynamics recorded in four SUTs for $195 \mathrm{~mW} \mathrm{CW}$ exposure for $90 \mathrm{~min}$. At the initial stage (during the first $8 \mathrm{~s}$ ) all curves demonstrate similar dynamics without notable differences (Fig. 5b), confirming the results for SAR presented in the previous sections. After about $70 \mathrm{~s}$ of exposure, a crest (temperature peak) appears in the water sample (Fig. 5c). This moment precedes the initiation of convection in bulk [Kaviany, 1984; Tanasawa, 1995; Kohl et al., 2008]. At MMW frequencies, a similar effect was observed by Khizhnyak and Ziskin [1996] during exposure of 0.5-3.0-mm-thick layers of $\mathrm{NaCl}$ solution, in the $53-78 \mathrm{GHz}$ range at $\mathrm{SAR}$ levels up to $80 \mathrm{~kW} / \mathrm{kg}$. For exposure durations 
exceeding $70 \mathrm{~s}$, the temperature rise in the liquid samples is noticeably lower than that in the agar gel due to convection.

No noticeable difference $(<1 \%)$ is observed between culture medium and culture medium with a cell monolayer, confirming that the presence of a cell layer practically does not change temperature rise in the culture medium.

Convection in water reduces the temperature rise by $3.34 \pm 0.12{ }^{\circ} \mathrm{C}(30 \pm 3 \%)$ with respect to the agar gel at steady state (mean \pm SD in five independent recordings).

The temperature rise in the culture medium is higher than in water by $0.7{ }^{\circ} \mathrm{C}(8 \pm 2 \%)$. However, the difference in SAR between culture medium with and without cells is only $2 \%$ and $1 \%$, respectively, when compared to water (Table 3 ). The latter is related to the difference between the electrical conductivities $\sigma$ of water and culture medium (Table 1). The remaining difference can be attributed to different 1) heat capacity (i.e., a higher power should be delivered to water to obtain the same temperature increment as in the culture medium), and 2) viscosity as discussed hereafter.

Natural convection may be predicted by using Rayleigh and Grashof numbers. The Ra number is expressed as follows:

$$
R a=\frac{g \beta x^{3} \Delta T}{v \alpha}
$$

where $g$ is the gravitational acceleration, $\beta$ is the coefficient of volume expansion, $x$ is the characteristic length of the geometry, $\Delta T$ is a driving temperature difference between the temperature of the surface and fluid sufficiently far from the surface, $v$ is kinematic viscosity of the fluid, and $\alpha$ is the thermal diffusivity equal to $k / \rho \cdot C$, i.e., the thermal conductivity divided by density and specific heat capacity of liquid [Bird et al., 1960]. As convection was initiated in water when its volume exceeded $1.5 \mathrm{ml}$ at $\Delta T \approx 3{ }^{\circ} \mathrm{C}$ (see sub-section (c) Role of liquid volume), the critical Ra number was calculated using the parameters given in Table 4 
and $x=2.9 \cdot 10^{-3} \mathrm{~m}$ at $\Delta T=3{ }^{\circ} \mathrm{C}$. The height of $1.5 \mathrm{ml}$ of water in the center of a well was measured experimentally. It was different from the calculated one $\left(3.9 \cdot 10^{-3} \mathrm{~m}\right)$ due to the formation of a meniscus. For considered MMW exposure conditions, the critical Ra number of water was found to be equal to $2.3 \cdot 10^{4}$. In $2 \mathrm{ml}$ (i.e., $x=5.2 \cdot 10^{-3} \mathrm{~m}$ ) of water typically used in the experiments, $\mathrm{Ra}=1.3 \cdot 10^{5}$ at $\Delta T=3{ }^{\circ} \mathrm{C}$. This number substantially exceeds the critical Ra number, indicating the occurrence of the natural convection in fluids during MMW exposure used in the experiments.

Grashof number is expressed as:

$$
G r=\frac{g \beta x^{3} \Delta T}{v^{2}}
$$

where designations of $g, \beta, x, \Delta T$, and $v$ are the same as those used for the Ra number. The $\mathrm{Gr}$ number was calculated using values provided in Table 4 and $x=5.2 \cdot 10^{-3} \mathrm{~m}$. It was found that $\mathrm{Gr}=3.1 \cdot 10^{4}$ and $1.3 \cdot 10^{5}$ for water, and $\mathrm{Gr}=2.8 \cdot 10^{4}$ and $1.15 \cdot 10^{5}$ for culture medium, at $\Delta T=$ $3{ }^{\circ} \mathrm{C}$ and $\Delta T=12.5^{\circ} \mathrm{C}$, respectively. The values of the $\mathrm{Gr}$ number assume that there is a laminar flow in both fluids $\left(\mathrm{Gr}<1 \cdot 10^{9}\right)$. It is worth mentioning that $\Delta T$ elevation increases convection of fluid. The $\Delta T$ elevation from 3 to $12.5^{\circ} \mathrm{C}$ increases the $\mathrm{Gr}$ number by 4.2 times. The greater the Gr number, the greater the fluid movement or convection. The Gr numbers for culture medium are about 10 and $13 \%$ lower than those for water at $\Delta T=3{ }^{\circ} \mathrm{C}$ and $\Delta T=12.5$ ${ }^{\circ} \mathrm{C}$, respectively.

Viscosity is the only different parameter between water and culture medium that affects the Gr number. Therefore, the higher viscosity of the culture medium would lead to less convection than water under the same exposure conditions. This, together with a $2 \%$ difference in SAR, explains higher temperature rise in the culture medium. Indeed, convection transports heat away from the micro TC located at the bottom of the well, reducing the measured temperature. 


\section{b. Dependence on SAR}

Initiation of convection in the liquid samples was investigated as a function of SAR in 1.8 to $24 \mathrm{~kW} / \mathrm{kg}$ range. We considered only water and culture medium without cells since the culture medium with cells demonstrated temperature dynamics similar to the culture medium without cells (Fig. 5a). Figure 6 shows that a temperature peak is generated in the water sample at $\mathrm{SAR} \geq 7.8 \mathrm{~kW} / \mathrm{kg}$. In the culture medium, the peak appears only at $\mathrm{SAR}=$ $24 \mathrm{~kW} / \mathrm{kg}$. This can be attributed to the higher viscosity of the culture medium compared to water. The occurrence of convection is facilitated by the higher SAR [Antohe and Lage, 1996].

The time point at which the peak appears depends on the SAR. For instance, in water at $7.8 \mathrm{~kW} / \mathrm{kg}$, convection starts after about one min, while at $24 \mathrm{~kW} / \mathrm{kg}$ it is triggered after $40 \mathrm{~s}$. Results are in agreement with the literature; previous studies have demonstrated that the onset of convection occurs earlier when the heat flux, which can be considered proportional to the SAR, increases [Jhaveri and Homsy, 1982; Kim and Kim, 1986; Yang and Choi, 2002]. Note that the product of the time point at which convection appears and the temperature reached at this time point remains constant $\left(56 \pm 5^{\circ} \mathrm{C} \cdot \mathrm{s}\right)$.

Figure 7 shows the difference between the temperature in the culture medium and water at steady state, $\Delta T_{C M-W}$, as a function of SAR. $\Delta T_{C M-W}$ is proportional to SAR, and $\Delta T_{C M-W}$ (SAR) can be interpolated using the linear relation, $\Delta T_{C M-W}=0.053 \cdot \mathrm{SAR}$, with $R=0.88$.

\section{c. Role of liquid volume}

The volume of a liquid sample represents an important parameter to consider when planning an in vitro experiment. We studied heating of the volume of liquid ranging from 0.5 to $4 \mathrm{ml}$ corresponding to 1.3 - and 10.4-mm height, respectively (Fig. 8). 
For $0.5 \mathrm{ml}$, the temperature rise is significantly higher than for volumes exceeding $1 \mathrm{ml}$. While the heat conduction mainly determines the temperature dynamics up to $1.5 \mathrm{ml}$, the effect of convection becomes pronounced when the volume exceeds $1.5 \mathrm{ml}$. The greater the volume, the earlier the temperature decrease is, due to convection (after $17 \mathrm{~s}, 14 \mathrm{~s}$, and $12 \mathrm{~s}$ for $2 \mathrm{ml}, 3 \mathrm{ml}$, and $4 \mathrm{ml}$, respectively). The difference between the maximum temperature in the liquid and agar gel also increases with the volume by $21 \%, 33 \%$, and $45 \%$, respectively.

Note that convection also affects the cooling dynamics, i.e., cooling is faster in larger volumes where the effect of convection is more pronounced.

The impact of the liquid volume on the variation of the Gr number was evaluated for $x$ ranging from $1.3 \cdot 10^{-3}$ to $10.4 \cdot 10^{-3} \mathrm{~m}$ (i.e., the liquid volumes used in experiments). The meniscus effect was not included in our calculations. The results of calculations obtained using equation (2) and parameters for $g, \beta, \Delta T$, and $v$ given in Table 4 are presented in Table 5. The increase of $\Delta T$ and $x$ results in elevation of Gr. It is worth noting that the liquid volume effect is stronger than the one related to the temperature change from 3 to $12.5^{\circ} \mathrm{C}$. The results show that an increase of the liquid volume from 0.5 to $4 \mathrm{ml}$ results in a rise of the Gr number of about three orders of magnitude, which explains why, by increasing the volume, the maximum temperature elevation was reduced and cooling was faster, i.e., the effect was due to increased convection.

These results suggest that the culture medium volume in in vitro experiments at MMW frequencies has to be carefully chosen due to convection, which affects the maximum temperature as well as cooling dynamics.

\section{PW induced heating}

\section{a. Heating in water and agar gel}


The electromagnetic pulse train consisted of $1.5 \mathrm{~s}$ square-wave amplitude-modulated MMW pulses with a peak power of $4.2 \mathrm{~W}$ and period of $20 \mathrm{~s}$. In the beginning of exposure, the heat pulses measured in the SUTs had a relative amplitude of $10^{\circ} \mathrm{C}$ (Fig. 9a). The volumes for both water and agar gel were set to $2 \mathrm{ml}$. The relative peak amplitude of pulses was reduced by $9 \%$ and $11 \%$ in water and agar gel, respectively, after 10 min of exposure (Fig. 9c,d). Then it remained almost constant until the end of exposure (Fig. 9b,c,d).

While the relative amplitude of the peak remains constant after $10 \mathrm{~min}$ of exposure for both samples, the cooling dynamics of samples with and without convection are different. For each pulse, they remain similar for about $3 \mathrm{~s}$ (Fig. 9a,b). Afterwards, convection in water results in a faster decrease of water temperature, leading to the maximum difference of $1.3{ }^{\circ} \mathrm{C}$ at the end of the cooling phase compared to the agar gel. This is responsible for the lower average temperature in the liquid compared to the agar gel (Figs. 9c,d).

The results suggest that for heat pulses, the main effect of convection is a decrease of the average temperature that results from the increased cooling rate in liquids compared to the agar gel.

\section{b. Dependence on pulse duration}

Initiation of convection was investigated as a function of the pulse duration $\tau$ ranging from 1 to $6.5 \mathrm{~s}$ (Fig. 10). For the constant peak power of $4.2 \mathrm{~W}$, the amplitude of the temperature pulses increases with the pulse duration from $8(1 \mathrm{~s}$ pulse $)$ to $21.4{ }^{\circ} \mathrm{C}(6.5 \mathrm{~s}$ pulse $)$ and from 8 (1 s pulse) to $25^{\circ} \mathrm{C}(6.5 \mathrm{~s}$ pulse) in water and gel, respectively. When convection is absent ( $\tau$ $=1 \mathrm{~s}$ ), the shape of the temperature pulses for both samples is identical. For $\tau=1.5$, the amplitude of the temperature pulse remains the same. However, the cooling rate in the agar gel is slightly faster in the time interval from 0 to $3 \mathrm{~s}$ after exposure, and afterwards it becomes slower compared to that in the liquid. 
For $\tau=2$ to $4 \mathrm{~s}$, the amplitude of the temperature pulse generated in water is higher than the one in agar gel. The maximum difference $\left(2.13 \pm 0.57^{\circ} \mathrm{C}\right)$ occurs for $\tau=3 \mathrm{~s}$. We presume that this phenomenon is of the same nature as for the temperature peak presented in Figure 5c. The time difference of appearance of this peak at CW (70 s) and PW (3 s) exposures is caused by the difference in power (195 $\mathrm{mW}$ for $\mathrm{CW}$ and $4.2 \mathrm{~W}$ for PW).

For $\tau \geq 4.5 \mathrm{~s}$, convection strongly changes the heat pulse shape, decreasing the peak temperature. In addition, it increases the cooling rate from 1.8 to $7.12{ }^{\circ} \mathrm{C} / \mathrm{s}$ when pulse duration increases from 1 to $6.5 \mathrm{~s}$ (here the cooling rate is averaged over the initial cooling phase until the temperature drops to $\left.\Delta T_{\max } / 2\right)$.

These results suggest that, for a given power, changes in the pulse shape due to convection depend on the pulse duration. Convective currents during the warm-up phase can be avoided or reduced by decreasing the pulse duration. Note that pulse duration is proportional to the total absorbed power during a pulse. Detailed investigation of the temperature dynamics induced by short- and high-amplitude MMW pulses would require more powerful generators along with multi-physical modeling.

\section{Conclusion}

In this study, we analyzed the effect of convection on temperature dynamics in models representing a typical in vitro exposure scenario at MMW frequencies in CW and PW regimes. Temperature dynamics in four different samples were investigated: 1) a cell monolayer attached to the bottom of a 12-well TCP and covered by the culture medium, 2) culture medium without cells, 3) distilled water, and 4) a convection-free medium with electromagnetic and thermodynamic properties close to water (agar gel). 
Previous numerical studies have demonstrated that the presence of a thin cell monolayer only slightly perturbs the power absorption in in vitro samples at MMW frequencies. We experimentally demonstrated that a cell monolayer practically does not change heating dynamics at the considered exposure intensities. This suggests that when a TC is used to monitor the local temperature dynamics with a microscale resolution, the measurements can be performed in a separate well without cells to avoid damaging and contaminating cells.

For CW exposure, a temperature peak appears after several tens of seconds, preceding initiation of convection in bulk. The time point at which the peak appears depends on SAR. The higher the SAR, the earlier the peak appears, triggering convection. It is more pronounced in free water compared to the culture medium due to the lower viscosity of water. This effect has to be carefully taken into account in in vitro bioelectromagnetic experiments as it may affect the biological outcome and result in a different cellular response compared to conventional heating used as a control.

Our results also indicate that the liquid volume is an important parameter influencing convection. Its effect is often ignored or underestimated in in vitro experiments. Indeed, the convection is triggered earlier when the volume increases. Increasing the volume also results in a greater cooling effect, influencing temperature dynamics at the cellular level. Furthermore, it also affects cooling dynamics after exposure is switched off. Specifically, cooling is faster in larger volumes.

In PW regime, convection strongly depends on the pulse duration. For the exposures in these experiments, at $\tau=1 \mathrm{~s}$, the shape of the thermal pulse is not impacted by convection. For $\tau=1.5$, the amplitude of the thermal pulses remains the same. However, cooling dynamics are slightly affected by convection, resulting in a decrease of the average temperature for prolonged exposures. For $\tau=2$ to $4 \mathrm{~s}$, convection increases the amplitude of the thermal pulse in liquid, and this effect is the most pronounced for $\tau=3 \mathrm{~s}$ for the 
considered exposure condition. The exact understanding of the mechanism of the observed effect would require analysis of convective currents in $3 \mathrm{D}$, which is not achievable using the techniques employed in this study. Detailed analysis of this effect is a future aim of our group. For $\tau \geq 4.5 \mathrm{~s}$, convection strongly changes the heat pulse shape, decreasing the peak temperature and increasing the cooling rate.

Note that heating was studied under the conditions of local heating with narrow beam using an open-ended WG. When samples are exposed with broader-beam antennas, larger areas of uniform heating at the bottom of the well can occur. In this case, the boundaries for convection will also change, potentially affecting the heating.

In conclusion, our results suggest that in planning in vitro studies in the MMW band, convection is one of the parameters that has to be carefully taken into account. It may affect cellular response through indirect mechanisms, such as the local change of the concentration of oxygen or nutrients transported by the culture medium. Deeper investigation of convection mechanisms requires numerical analysis of the multi-physics problem, which constitutes one of the perspectives of a future study.

\section{References}

Alekseev SI, Zhadobov MV, Fesenko EE Jr, Fesenko EE. 2017. Millimeter wave dosimetry at exposure of cell monolayer. Biophysics 62(2):261-264.

Alekseev SI, Ziskin MC. 2001. Distortion of millimeter-wave absorption in biological media due to presence of thermocouples and other objects. IEEE Trans Biomed Eng 48(9):1013-1019.

Alekseev SI, Ziskin MC. 2003. Local heating of human skin by millimeter waves: a kinetics study. Bioelectromagnetics 24(8):571-581.

Alekseev SI, Ziskin MC, Fesenko EE. 2011. Problem using a thermocouple for measurements of skin temperature rise during the exposure to millimeter waves. Biophysics 56(3):525-528. 
Antohe BV, Lage JL. 1996. Amplitude effect on convection induced by time-periodic horizontal heating. Int J Heat Mass Transfer 39(6):1121-1133.

Ayappa KG, Brandon S, Derby JJ, Davis HT, Davis EA. 1994. Microwave driven convection in a square cavity. AiChE J 40:1268-72.

Bedoya M, Del Rio AM, Chiang J, Brace CL. 2014. Microwave ablation energy delivery: influence of power pulsing on ablation results in an ex vivo and in vivo liver model. Med Phys 41(12):123301.

Bejan A. 2013. Convection Heat Transfer, fourth edition. New York, NY: John Wiley and Sons, pp $168-294$.

Betskii OV, Devyatkov ND, Kislov VV. 2000. Low intensity millimeter waves in medicine and biology. Crit Rev Biomed Eng 28(1-2):247-268.

Bird RB, Stewart WE, Lightfoot EN. 1960. Transport Phenomena. New York, NY: John Wiley and Sons, pp 243-309.

Boriskin AV, Zhadobov M, Steshenko S, Le Dréan Y, Le Coq L, Person C, Sauleau R. 2013. Enhancing exposure efficiency and uniformity using a choke ring antenna: application to bioelectromagnetic studies at $60 \mathrm{GHz}$. IEEE Trans Microwave Theory Tech 61(5):2005-2014.

Cengel YA. 2004. Heat Transfer: A Practical Approach, second edition. Boston, MA: McGraw-Hill, pp 333-360.

Cetas TC. 1982. Thermometry. In: Lehmann JF, editor. Therapeutic Heat and Cold. Third edition. Baltimore, MD: Williams and Wilkins, pp 35-69.

Chahat N, Zhadobov M, Sauleau R, Alekseev SI. 2012. New method for determining dielectric properties of skin phantoms at millimeter waves based on heating kinetics. IEEE Trans Microwave Theory Tech 60(3):827-832.

Chapman AJ. 1967. Heat Transfer, second edition. New York, NY: Macmillan Company, pp 356-370. Constable RT, Dunscombe PB, Tsoukatos A, Malaker K. 1987. Perturbation of the temperature distribution in microwave irradiated tissue due to the presence of metallic thermometers. Med Phys 14:385-388. 
Cueille L, Collin A, Pivain C, Leveque P. 2008. Development of a numerical model connecting electromagnetism, thermal and hydrodynamics to analyse in vitro exposure system. Ann Telecommun 63:17-28.

Dunscombe PB, Constable RT, McLellan J. 1988. Minimizing the self-heating artifacts due to the microwave irradiation of thermocouples. Int J Hyperthermia 4:437-445.

Dunscombe PB, McLellan J, Malaker K. 1986. Heat production in microwave-irradiated thermocouples. Med Phys 13:457-461.

Ellison WJ. 2007. Permittivity of pure water, at standard atmospheric pressure, over the frequency range $0-25 \mathrm{THz}$ and the temperature range $0-100^{\circ} \mathrm{C}$. J Phys Chem Ref Data 36(1):1-18.

Erdogdu F. 2008. Optimization in Food Engineering. Boca Raton, FL: CRC Press, p 416.

Fernández E, López D, Mijangos C, Duskova-Smrckova M, Ilavsky M, Dusek K. 2008. Rheological and thermal properties of agarose aqueous solutions and hydrogels. J Polym Sci B Polym Phys $46: 322-328$.

Fröhlich E, Bonstingl G, Höfler A, Meindl C, Leitinger G, Pieber TR, Roblegg E. 2013. Comparison of two in vitro systems to assess cellular effects of nanoparticles-containing aerosols. Toxicol In Vitro 27(1):409-17.

Giard DJ, Aaronson SA, Todaro GJ, Paul Arnstein, Kersey JK, Dosik H, Parks WP. 1973. In vitro cultivation of human tumors: establishment of cell lines derived from a series of solid tumors. J Natl Cancer Inst 51(5):1417-1423.

Goodhew S, Griffiths R. 2004. Analysis of thermal- probe measurements using iterative method to give sample conductivity and diffusivity data. Appl Energy 77(2):205-223.

Guraliuc AR, Zhadobov M, De Sagazan O, Sauleau R. 2014. Solid phantom for body-centric propagation measurements at $60 \mathrm{GHz}$. IEEE Trans Microwave Theory Tech 62(6):1373-1380.

Guy AW, Chou CK, McDougall JA. 1999. A quarter of in vitro research: a new look at exposure methods. Bioelectromagntics 20:21-39.

Habash RW, Bansal R, Krewski D, Alhafid HT. 2006. Thermal therapy, part 1: an introduction to thermal therapy. Crit Rev Biomed Eng 34(6):459-489. 
Hahn GM. 1974. Metabolic aspects of the role of hyperthermia in mammalian cell inactivation and their possible relevance to cancer treatment. Cancer Res 34(11):3117-3123.

Hancock CP, Dharmasiri N, White M, Goodman AM. 2013. The design and development of an integrated multi-functional microwave antenna structure for biological applications. IEEE Trans Microw Theory Tech 61(5):2230-2241.

He X, Bischof JC. 2003. Quantification of temperature and injury response in thermal therapy and cryosurgery. Crit Rev Biomed Eng 31(5-6):355-422.

Heitz WL, Westwater JW. 1971. Critical Rayleigh numbers for natural convection of water confined in square cells with L/D From 0.5 to 8 . J Heat Transfer 93(2):188-195.

Hodgson DA, Feldberg IB, Sharp N, Cronin N, Evans M, Hirschowitz L. 1999. Microwave endometrial ablation: development, clinical trials and outcomes at three years. Br J Obstet Gynaec 106(7):684-694.

Hunt JW, Lalonde R, Ginsberg H, Urchuk S, Worthington A. 1991. Rapid heating: critical theoretical assessment of thermal gradients found in hyperthermia treatments. Int J Hyperthermia 7(5):703718.

IAPWS (International Association for the Properties of Water and Steam). 2008. Release on the IAPWS Formulation 2008 for the viscosity of ordinary water substance, available at www.iapws.org [Last accessed 20 November 2018].

Incropera FP, DeWitt DP, Bergman TL, Lavine S. 2007. Fundamentals of Heat and Mass Transfer, sixth edition. New York, NY: John Wiley and Sons, pp 347-387 and 949-950.

Jeffreys H. 1926. The stability of a layer of fluid heated below. Lond Edinb Dubl Phil Mag 2(10):833-844.

Jhaveri BS, Homsy GM. 1982. The onset of convection in fluid layers heated rapidily in a timedependent manner. J Fluid Mech 114:251-260.

Kaviany M. 1984. Onset of thermal convection in a fluid layer subjected to transient heating from below. J Heat Transfer 106(4):817-823. 
Khizhnyak EP, Ziskin MC. 1996. Temperature oscillations in liquid media caused by continuous (nonmodulated) milli-meter wavelength electromagnetic irradiation. Bioelectromagnetics 17:223229.

Kohl MJ, Kristoffersen M, Kulacki FA. 2008. Stability and convection in impulsively heated porous layers. J Heat Transfer 130(11):112601-112609.

Kim CS, Hill RP, Kumaradas JC, Irvine R, Liu FF, Sherar MD. 1998. Effect of simultaneus pulsed hyperthermia and pulsed radiation treatment on survival of SiHa cells. Int J Hyperthermia 14(6):573-581.

Kim KH, Kim MU. 1986. The onset of natural convection in a fluid layer suddenly heated from the bottom. Int J Heat Mass Transfer 29(2):193-201.

Kojima M, Suzuki Y, Tsai C-Y, Sasaki K, Wake K, Watanabe S, Taki M, Kamimura Y, Hirata A, Sasaki K, Sasaki H. 2015. Characteristics of ocular temperature elevations after exposure to quasi- and millimeter waves (18-40 GHz). J Infrared Milli Terahz Waves 36:390-399.

Le Quément C, Nicolaz CN, Habauzit D, Zhadobov M, Sauleau R, Le Dréan Y. 2014. Impact of 60GHz millimeter waves and corresponding heat effect on endoplasmic reticulum stress sensor gene expression. Bioelectromagnetics 35(6):444-51.

Lienhard JH IV, Lienhard JH V. 1981. A Heat Transfer Textbook, fourth edition. Cambridge, MA: Phlogiston Press, pp 401-455.

Luyen H, Gao F, Hagness SC, Behdad N. 2014. Microwave ablation at $10.0 \mathrm{GHz}$ achieves comparable ablation zones to $1.9 \mathrm{GHz}$ in ex vivo bovine liver. IEEE Trans Biom Eng 61(6):1702-1710.

Mellala I, Oukaira A, Kengene E, Lakhssassi A. 2017. Thermal therapy modalities for cancer treatment: a review and future perspectives. Appl Sci Res Rev 4:14.

Paffi A, Liberti M, Apollonio F, Sheppard A, Balzano Q. 2015. In vitro exposure: linear and non-linear thermodynamic events in Petri dishes. Bioelectromagnetics 36:527-537.

Pickard WF, Straube WL, Moros EG, Fan X. 1999. Simplified model and measurements of specific absorption rate distribution in culture flask within a transverse electromagnetic mode exposure system. Bioelectromagnetics 20:183-193. 
Roti Roti JL. 2008. Cellular responses to hyperthermia (40-46 degrees C): cell killing and molecular events. Int J Hyperthermia 24(1):3-15.

Sandblom J, Theander S. 1991. The effect of microwave radiation on the stability and formation of gramicidin-A channels in lipid bilayer membranes. Bioelectromagnetics 12:9-20.

Sekins KM, Emery AF. 1982. Thermal Science for Physical Medicine. In: Lehmann JF, editor. Therapeutic Heat and Cold. Third edition. Baltimore, MD: Williams and Wilkins, pp 70-132.

Sharov VS, Kazarinov KD, Andreev VE, Putvinsky AV, Betsky OV. 1983. Acceleration of peroxidation of lipids exposed to electromagnetic radiation of millimeter wave range. Biophysics 28:146-147.

Sun DW. 2007. Computational Fluid Dynamics in Food Processing. Boca Raton, FL: CRC Press, pp $355-356$.

Tanasawa I. 1995. Experimental techniques in natural convection. Exp Therm Fluid Sci 10(4):503518.

Wessapan T, Rattanadecho P. 2014. Acqueous humor natural convection of the human eye induced by electromagnetic fields: in the supine position. J Med Biol Eng 3(4):251-258.

Yang DJ, Choi CK. 2002. The onset of thermal convection in a horizontal fluid layer heated from below with time-dependent heat flux. Phys Fluids 14(3):930-937.

Yoon J, Cho J, Kim N, Kim DD, Lee E, Cheon C, Kwon Y. 2011. High-frequency microwave ablation method for enhanced cancer treatment with minimized collateral damage. Int $\mathrm{J}$ Cancer 129(8):1970-1978.

Zhadobov M, Alekseev SI, Le Dréan Y, Sauleau R, Fesenko EE. 2015. Millimeter waves as a source of selective heating of skin. Bioelectromagnetics 36(6):464-475.

Zhadobov M, Alekseev SI, Sauleau R, Le Page Y, Le Dréan Y, Fesenko EE. 2017. Microscale temperature and SAR measurements in cell monolayer models exposed to millimeter waves. Bioelectromagnetics 38(1):11-21.

Zhadobov M, Augustine R, Sauleau R, Alekseev SI, Di Paola A, Le Quément C, Mahamoud YS, Le Dréan Y. 2012a. Complex permittivity of representative biological solutions in the 2-67 GHz range. Bioelectromagnetics 33(4):346-355. 
Zhadobov M, Sauleau R, Augustine R, Le Quément C, Le Dréan Y, Thouroude D. 2012b. Near-field dosimetry for in vitro exposure of human cells at $60 \mathrm{GHz}$. Bioelectromagnetics 33(1):55-64.

Zhadobov M, Sauleau R, Le Dréan Y, Alekseev SI, Ziskin MC. 2008. Numerical and experimental dosimetry for in vitro experiments. IEEE Trans Microwave Theory Tech 56(12):2998-3007.

Zhang M, Che Z, Chen J, Zhao H, Yang L, Zhong Z, Lu J. 2011. Experimental determination of thermal conductivity of water-agar gel at different concentrations and temperatures. J Chem Eng Data 56(4):859-864.

Zhang Q, Jackson TH, Ungan A. 2000. Numerical modeling of microwave induced natural convection. Int J Heat Mass Transfer 43(12):2141-2154.

Zhao JX. 2005. Numerical dosimetry for cells under millimetre-wave irradiation using Petri dish exposure set-ups. Phys Med Biol 50(14):3405-3421. 


\section{Figure captions}

Fig. 1. Schematic of the exposure system: exposure chamber (incubator), signal generation unit, and temperature recording instrumentation (dimensions are not to scale).

Fig. 2. CAD model of the considered exposure scenario. Zero of the coordinate system is located at the center of the lower surface of the well bottom.

Fig. 3. Computed SAR without TC (in black): a) at the interface with the bottom of the well in $\mathrm{E}$ (along $\mathrm{x}$ axis) and $\mathrm{H}$ (along $\mathrm{y}$ axis) planes, $\mathrm{b}$ ) along the well axis. Local increase of SAR in E plane due to TC is shown in gray.

Fig. 4. Temperature dynamics measured in water (a) and agar gel (b). Corresponding SAR values are reported in Table 3 . Black solid lines are the fitting curves.

Fig. 5. a) Temperature dynamics of exposure to $\mathrm{CW}$ at $195 \mathrm{~mW}$ in SUTs. SUTs' volume is 2 ml. b) Zoom within the first $8 \mathrm{~s}$ of exposure. c) Zoom within the first 10 min of exposure. Time point $\mathrm{t}=0$ corresponds to the beginning of exposure.

Fig. 6. Temperature history for different SAR conditions for a) water and b) culture medium. Time point $\mathrm{t}=0$ corresponds to the beginning of exposure.

Fig. 7. Difference between steady state temperature of the culture medium and water as a function of SAR. Experimental data points are represented by white circles. Each point corresponds to the difference between water and culture medium averaged over three measurements. Linear regression of these points is represented by the solid line. Dashed lines indicate the $95 \%$ confidence interval.

Fig. 8. Impact of the liquid volume on triggering convection, maximum heating, and cooling dynamics. The results were obtained for WG input power equal to $195 \mathrm{~mW}$. Liquid volumes were a) $0.5 \mathrm{ml}$, b) $1 \mathrm{ml}$, c) $1.5 \mathrm{ml}$, d) $2 \mathrm{ml}$, e) $3 \mathrm{ml}$, and f) $4 \mathrm{ml}$. 
Fig. 9. Temperature dynamics in PW regime in water (c) and agar gel (d). Black lines represent the average temperature calculated with $75 \mathrm{~s}$ span. Subplots on the top compare the dynamics in water and agar gel at the beginning (a) and at the end of exposure (b).

Fig. 10. Heating dynamics in water and agar gel for different pulse durations: a) $1 \mathrm{~s}, \mathrm{~b}) 1.5 \mathrm{~s}$, c) $2 \mathrm{~s}$, d) $2.5 \mathrm{~s}$, e) $3 \mathrm{~s}$, f) $3.5 \mathrm{~s}, \mathrm{~g}) 4 \mathrm{~s}$, h) $4.5 \mathrm{~s}$, i) $5 \mathrm{~s}$, j) $5.5 \mathrm{~s}, \mathrm{k}) 6 \mathrm{~s}$, and l) $6.5 \mathrm{~s}$. 


\section{Table captions}

Table 1. Relative permittivity $\varepsilon$ and conductivity $\sigma$ of considered materials.

Table 2. Thermal and mechanical properties of water, culture medium, and agar gel at $32{ }^{\circ} \mathrm{C}$.

Table 3. Computed (without TC) and measured peak values of SAR.

Table 4. Parameters used for calculations of the Ra and Gr numbers.

Table 5. Dependence of the Gr number on the temperature difference $\Delta T$ and height of liquid $x$. 


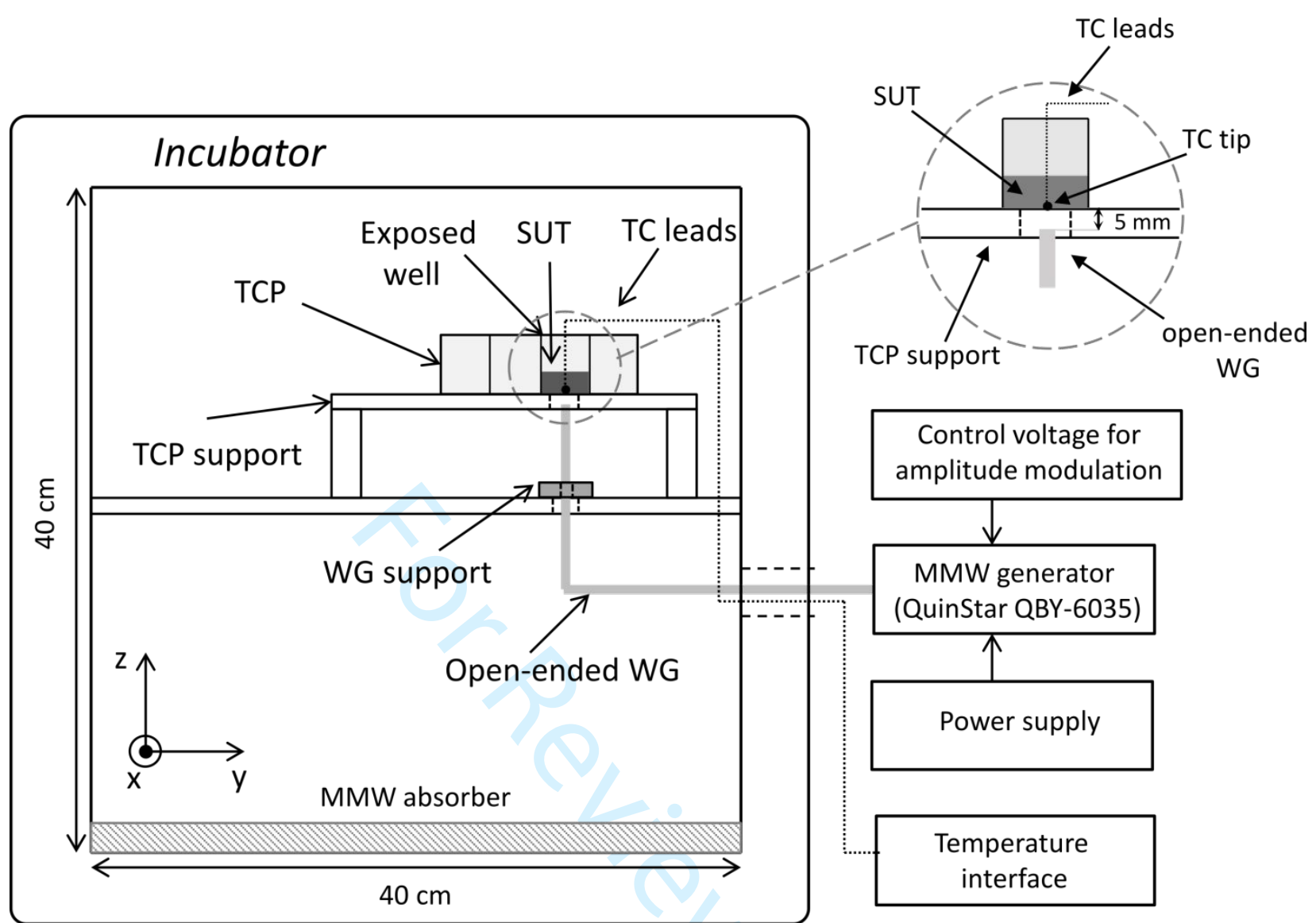

Fig. 1. 


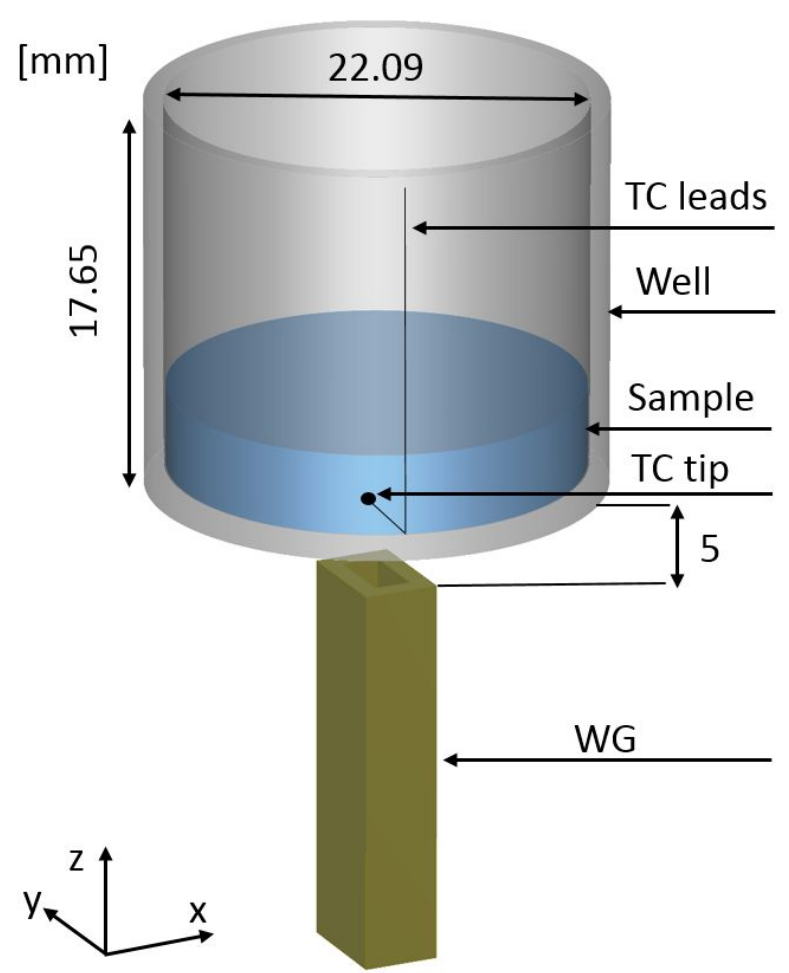

Fig. 2. 


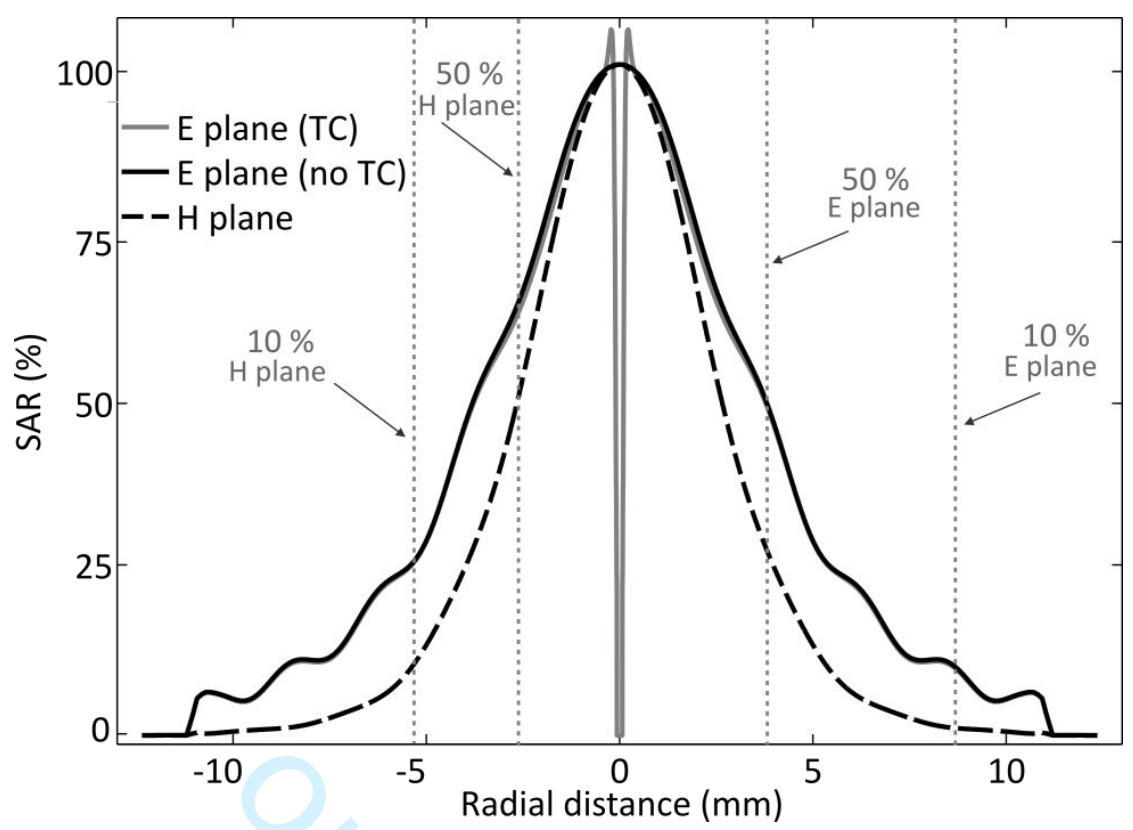

a)

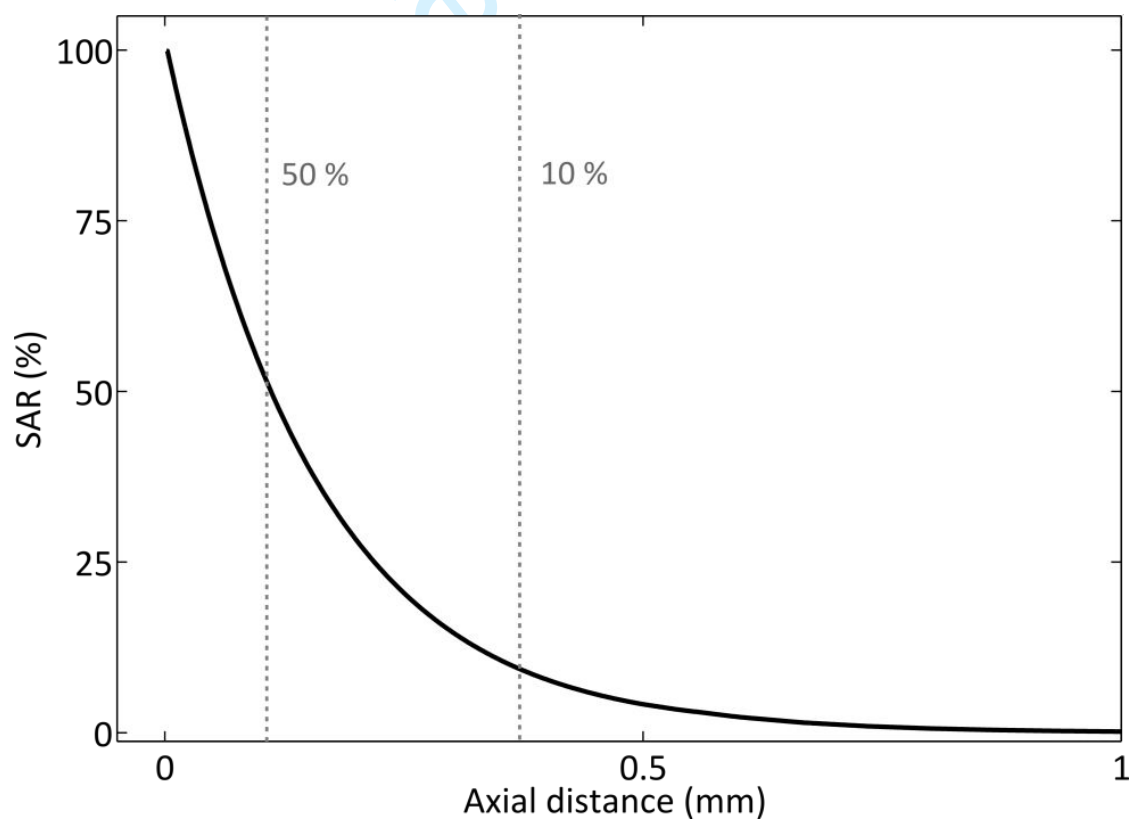

b)

Fig. 3. 


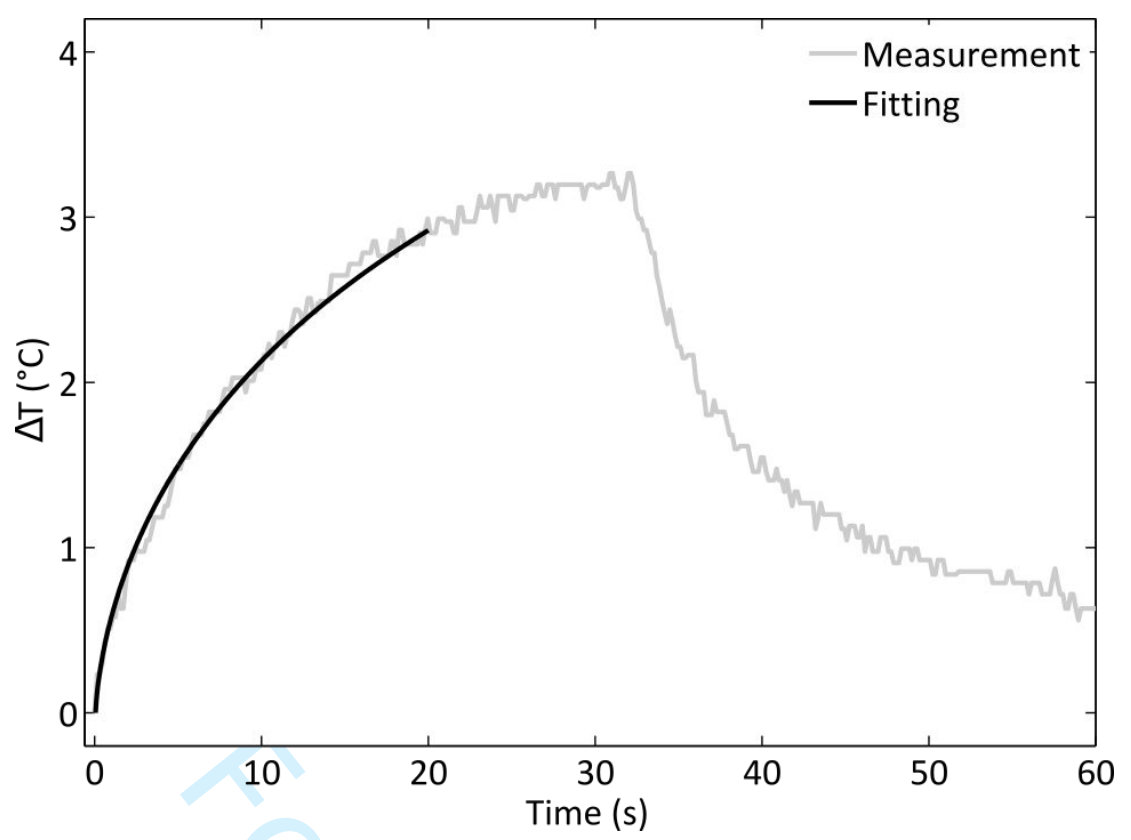

a)

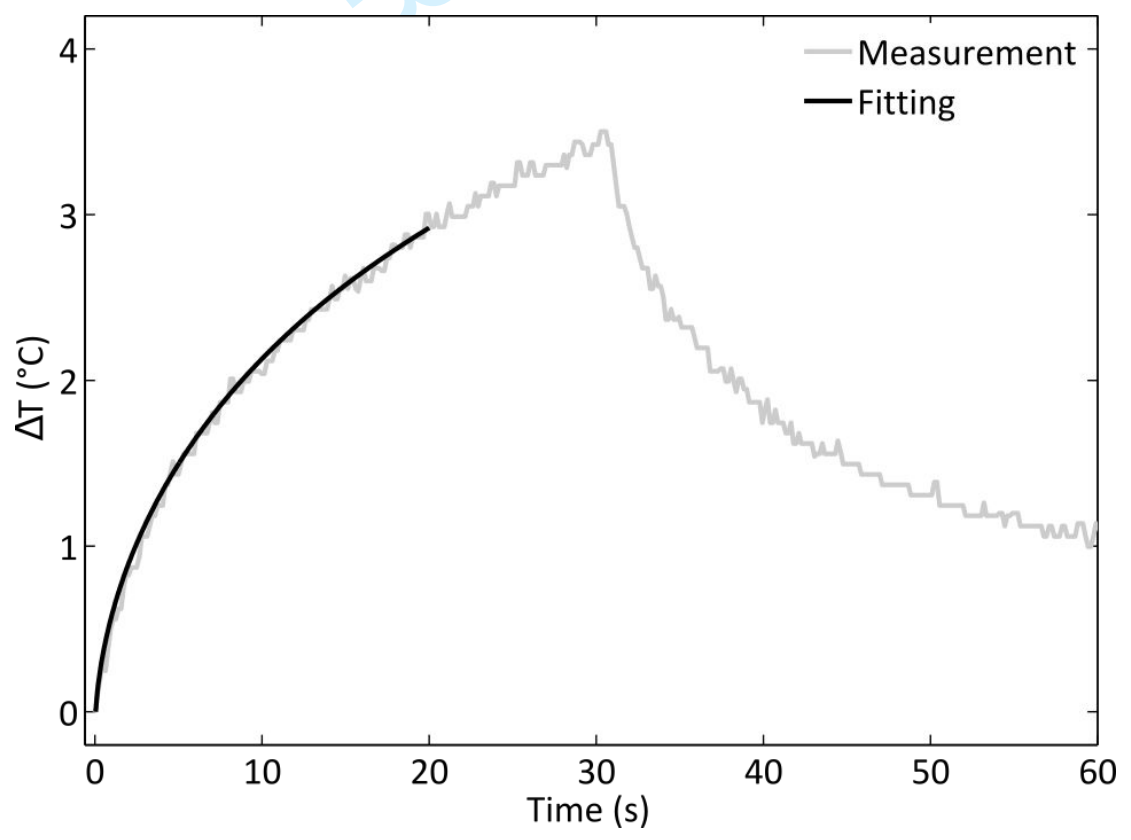

b)

Fig. 4. 


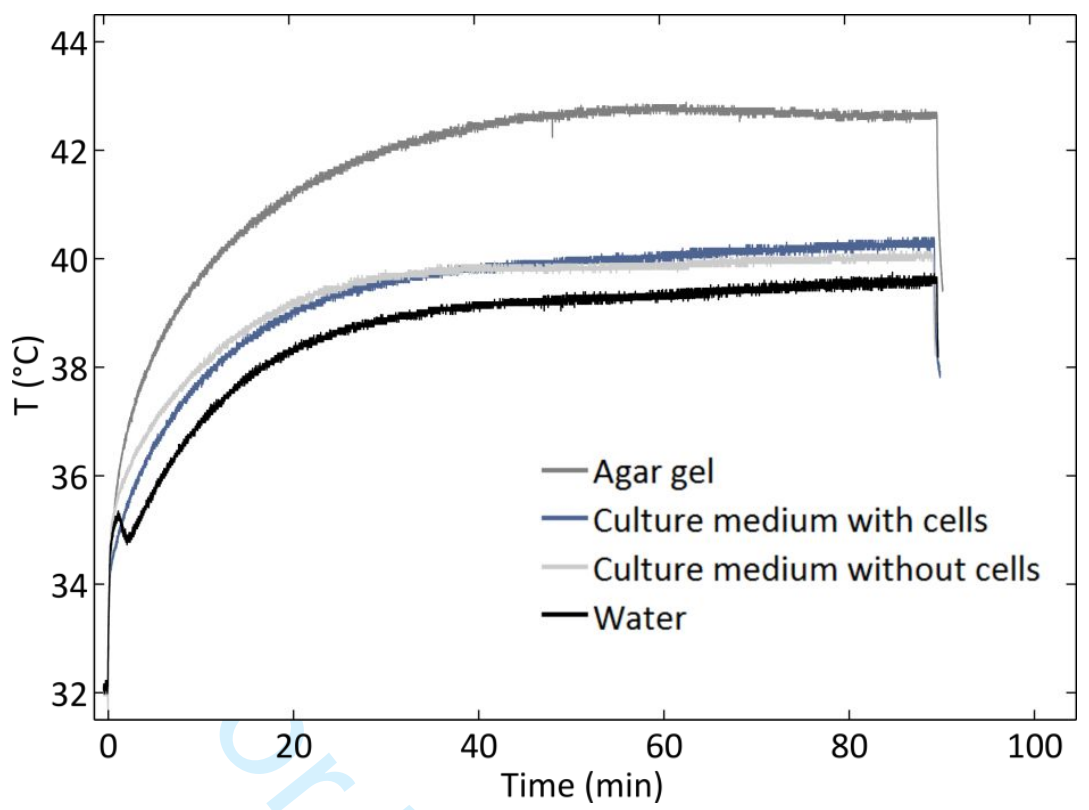

a)

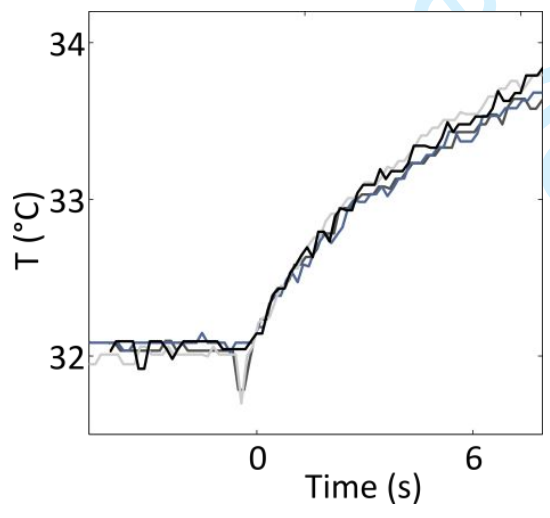

b)

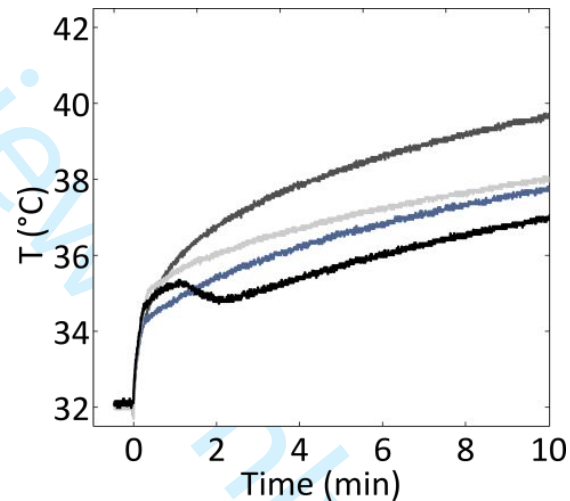

c)

Fig. 5. 


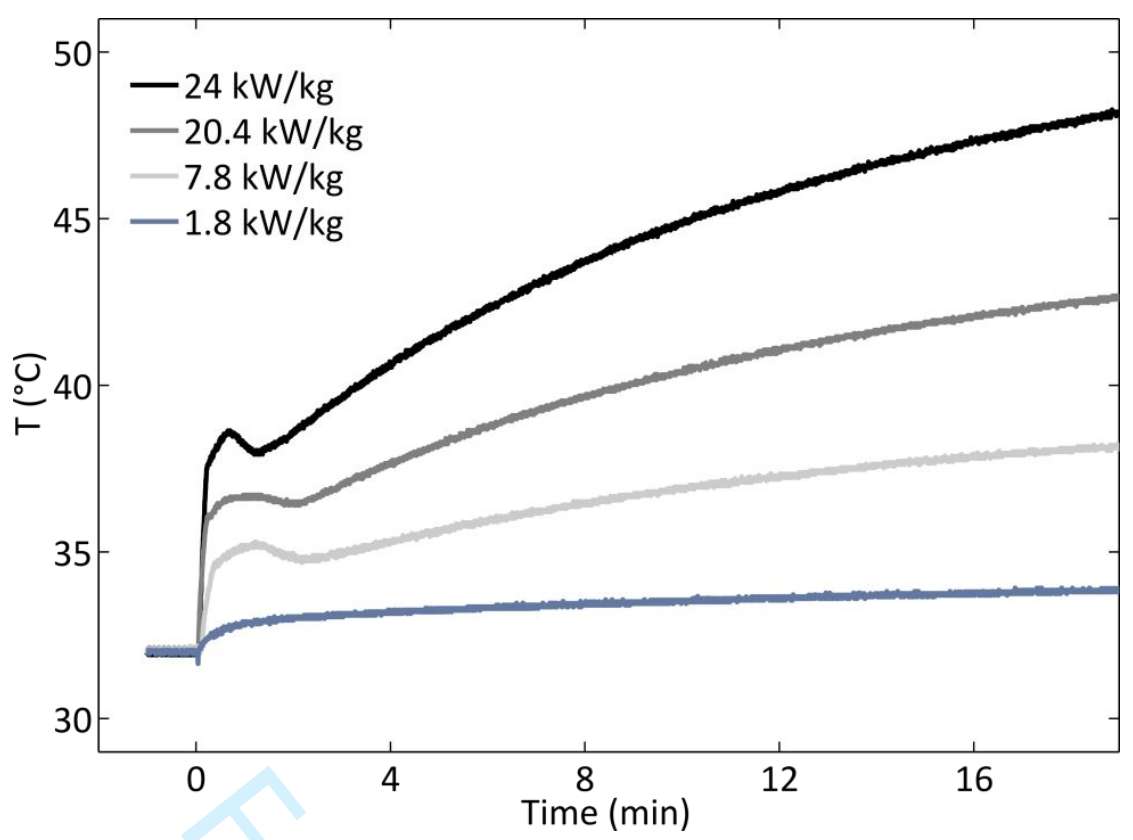

a)

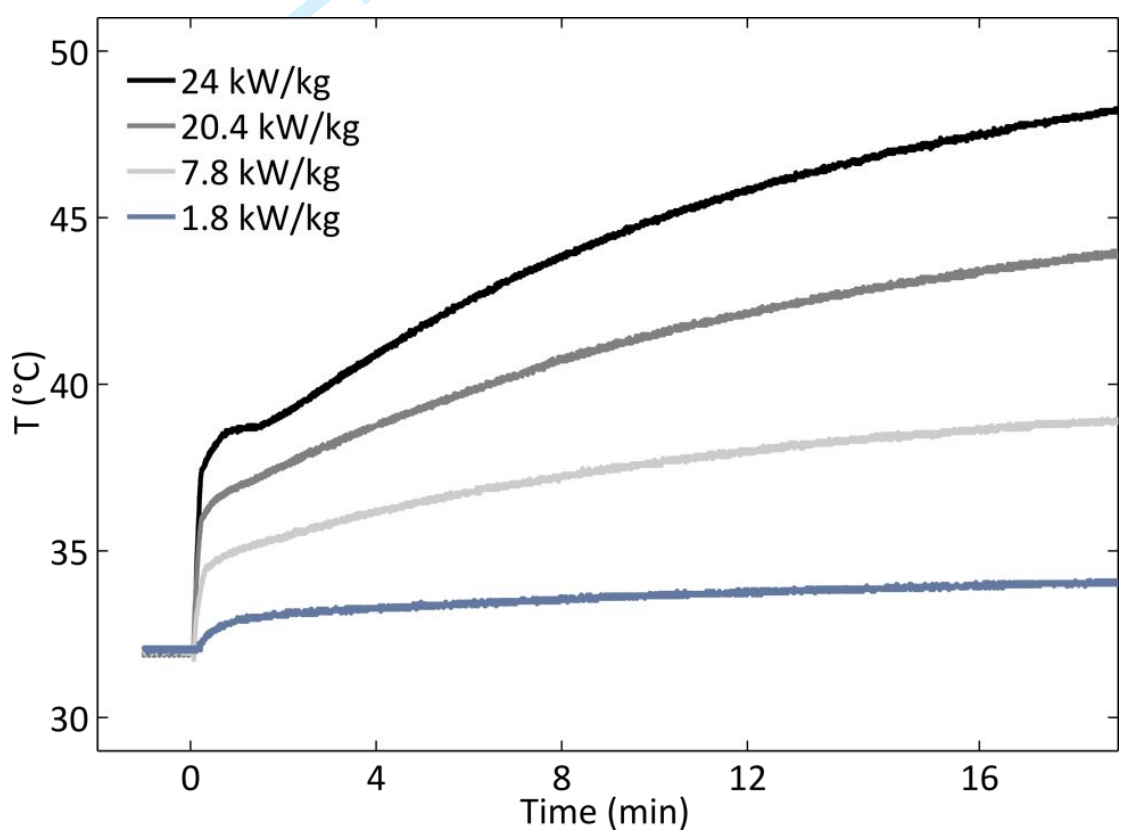

b)

Fig. 6. 


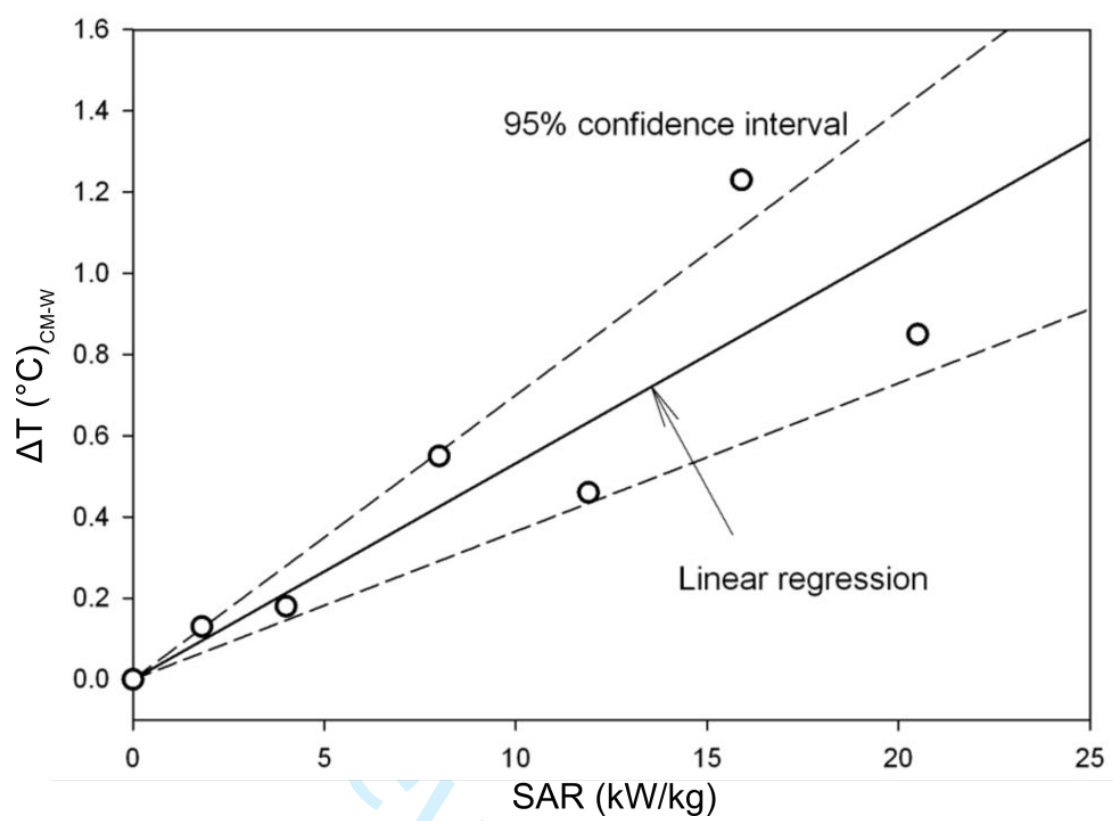

Fig. 7.

John Wiley \& Sons 

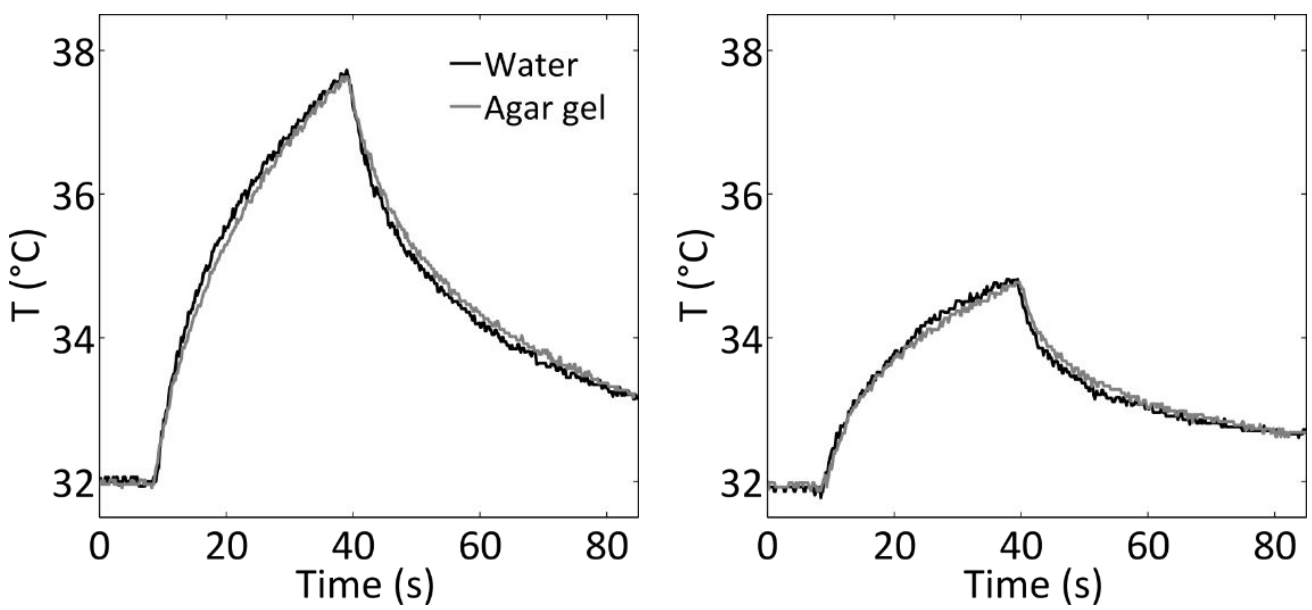

a) $0.5 \mathrm{ml}$
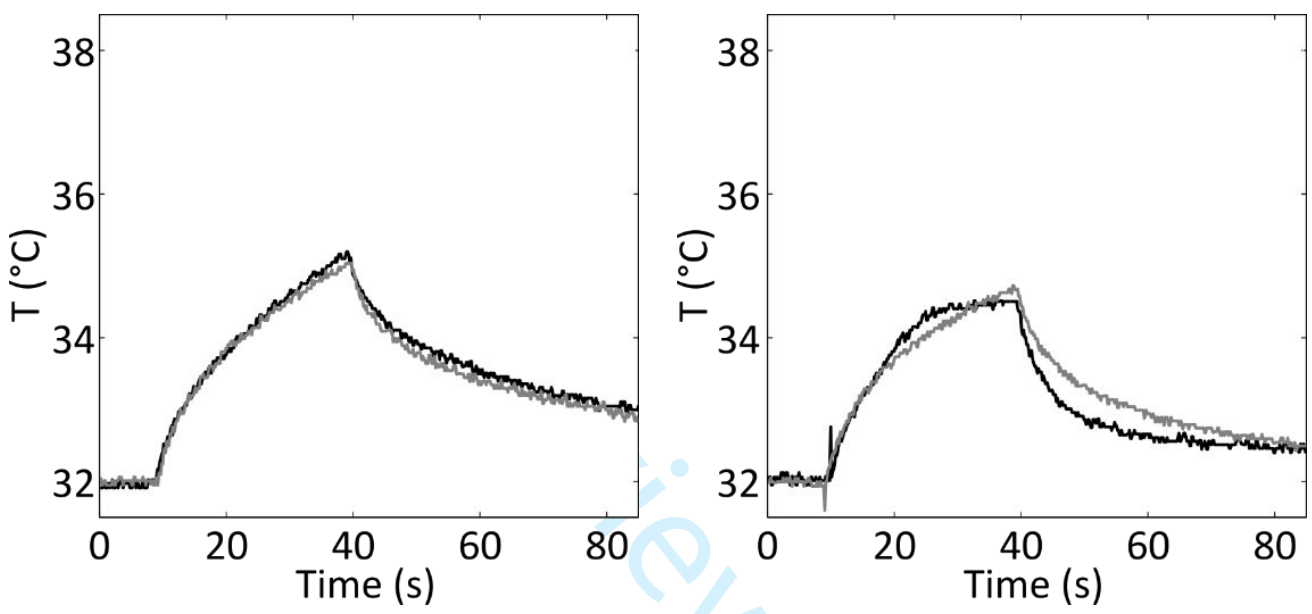

c) $1.5 \mathrm{ml}$

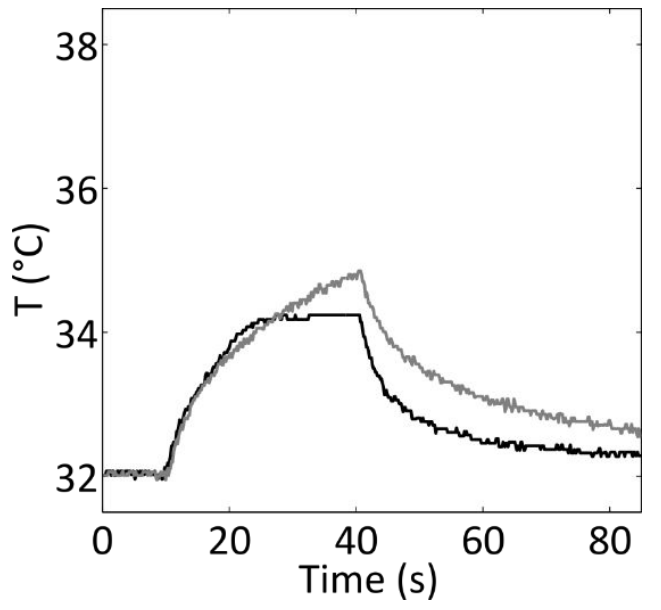

d) $2 \mathrm{ml}$

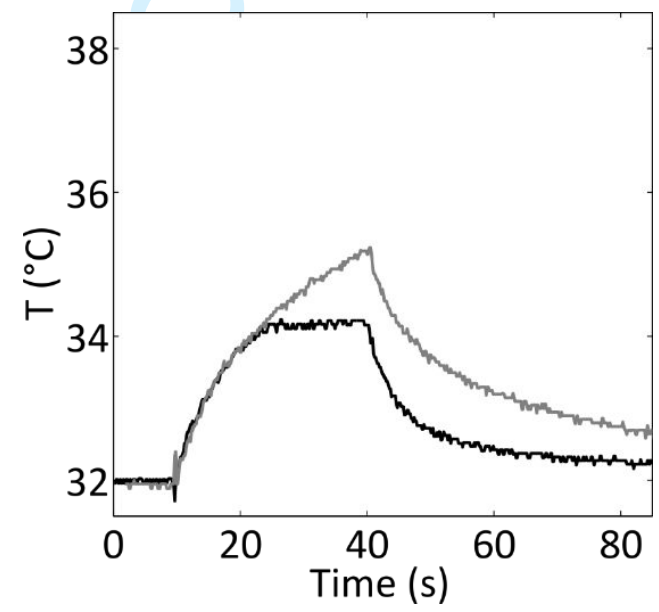

e) $3 \mathrm{ml}$

Fig. 8. 


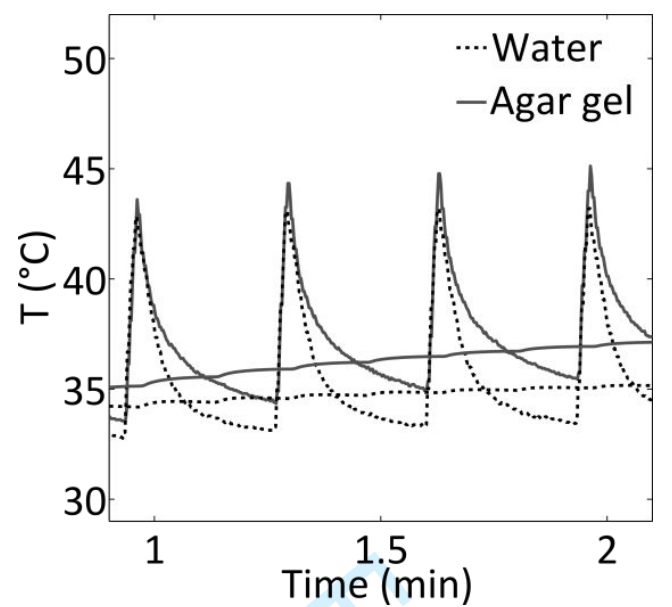

a)

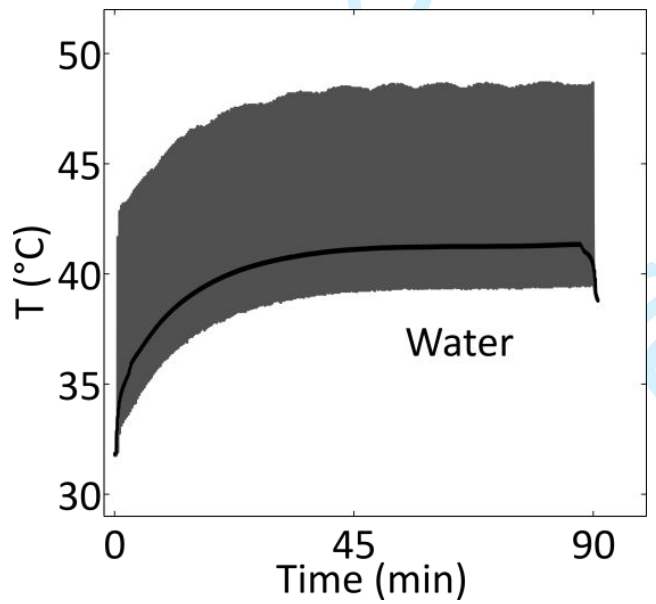

c)

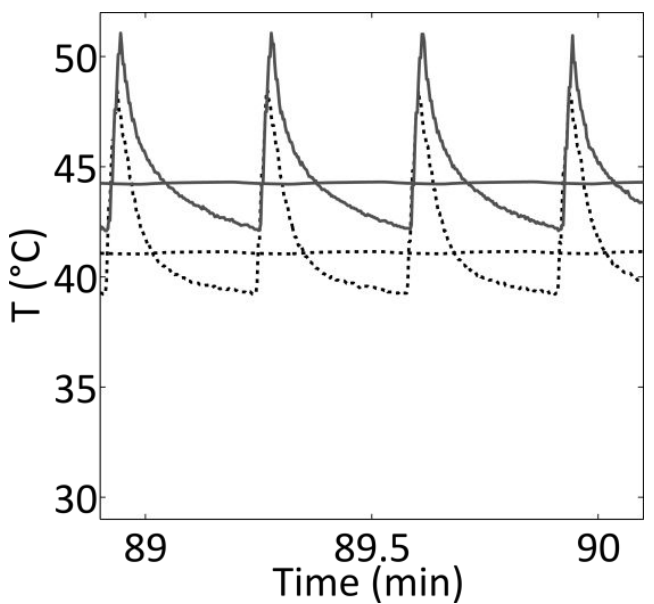

b)

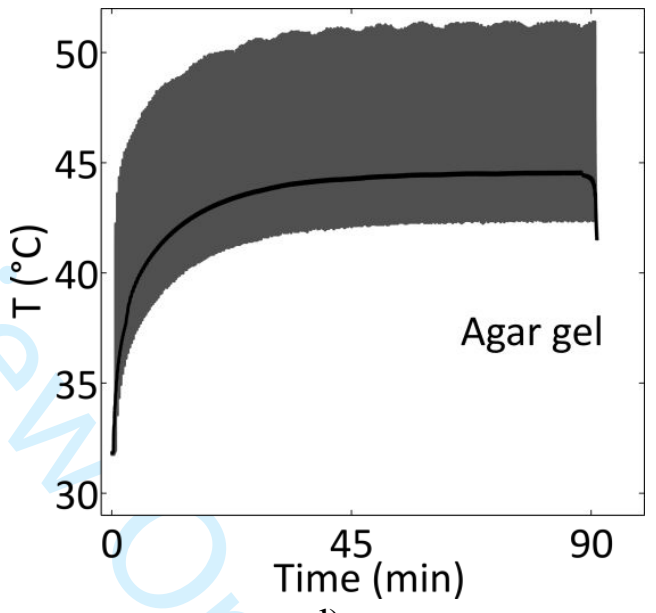

d)

Fig. 9. 

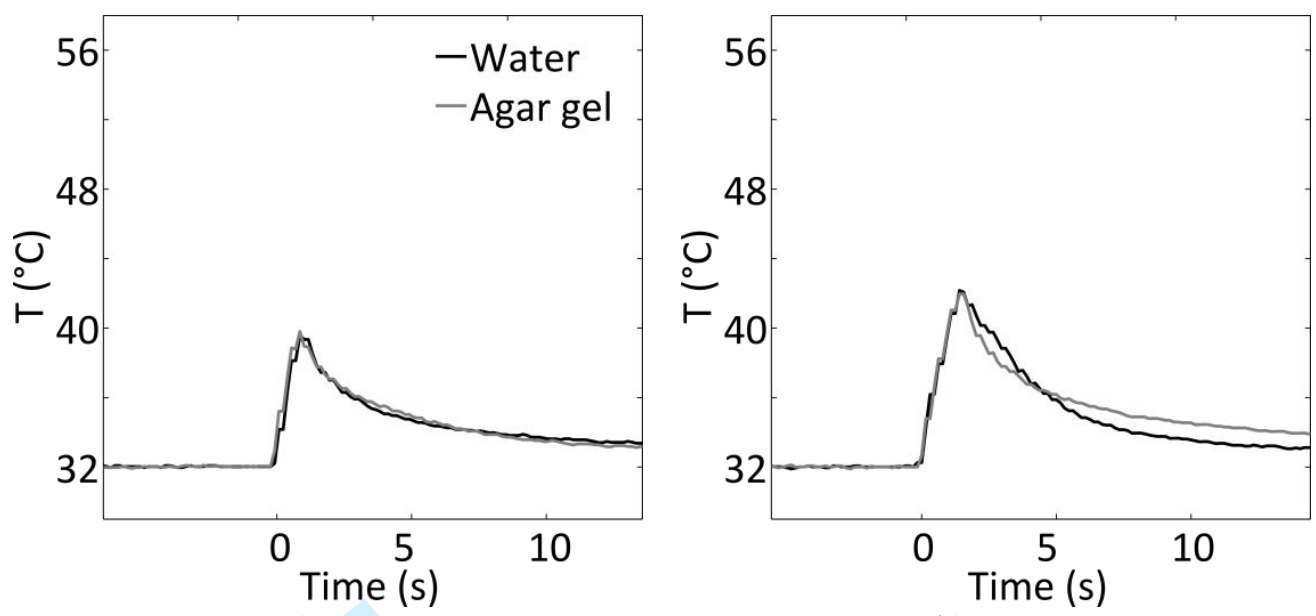

a) $1 \mathrm{~s}$
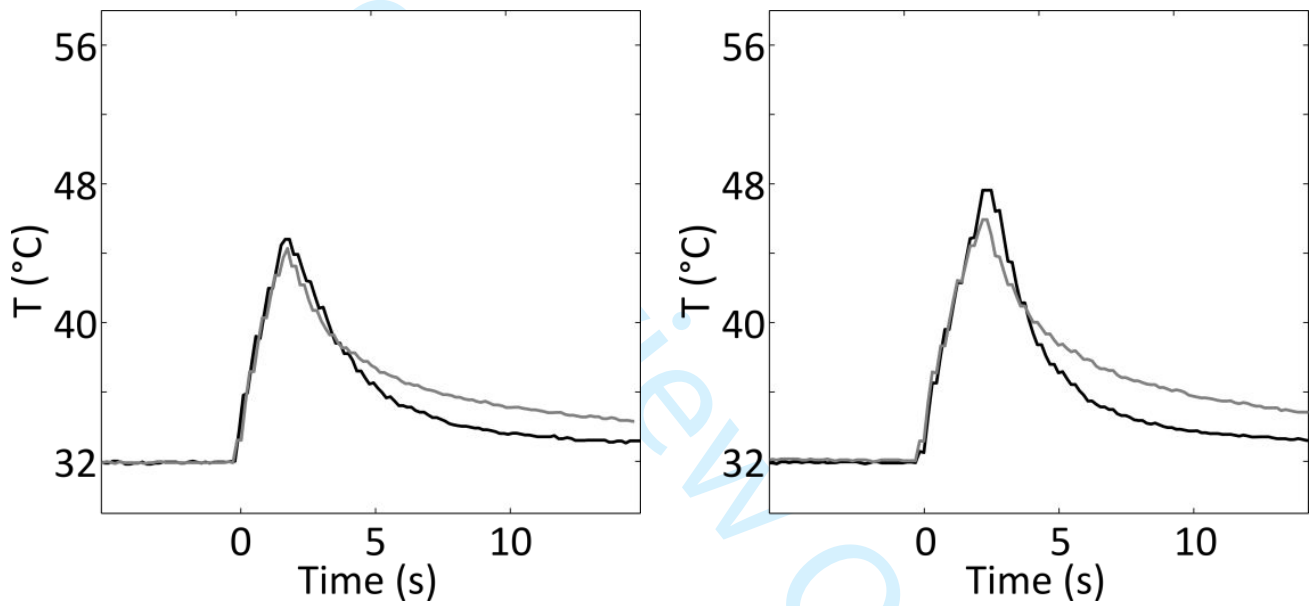

c) $2 \mathrm{~s}$
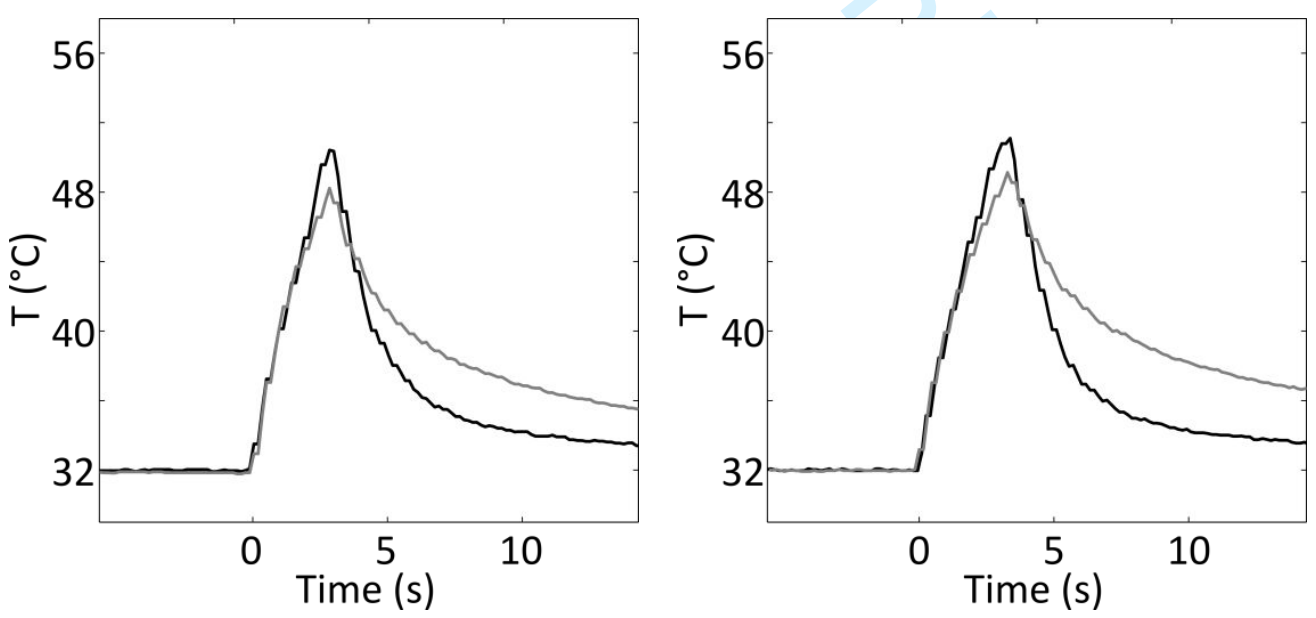

e) $3 \mathrm{~s}$

f) $3.5 \mathrm{~s}$ 

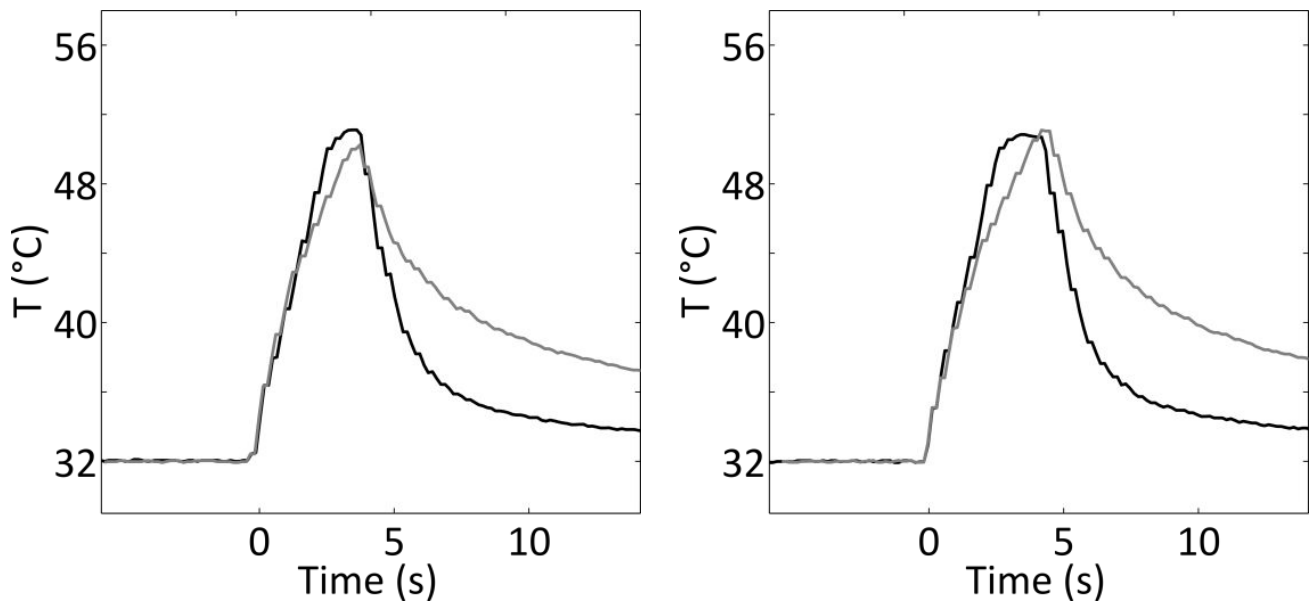

g) $4 \mathrm{~s}$

h) $4.5 \mathrm{~s}$
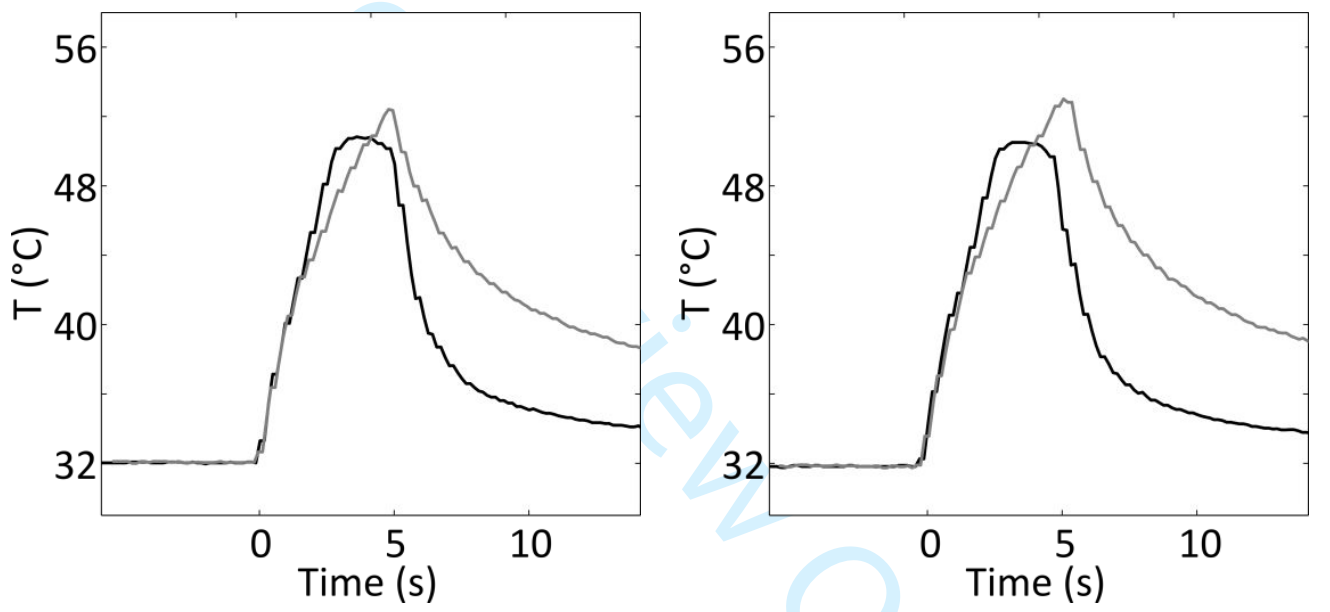

i) $5 \mathrm{~s}$
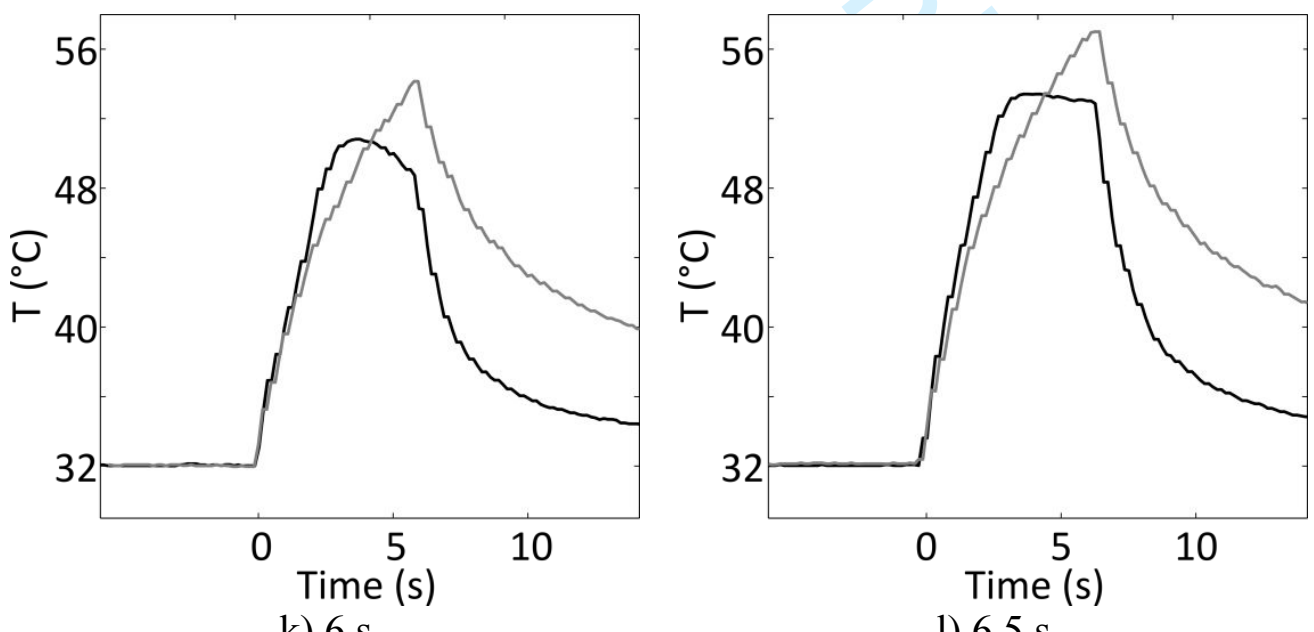

Fig. 10. 


\begin{tabular}{ccc}
\hline & $\varepsilon$ & $\sigma(\mathrm{S} / \mathrm{m})$ \\
\hline Water & 12.67 & 66.79 \\
Culture medium & 11.25 & 64.16 \\
Agar gel & 12.29 & 64.94 \\
Polystyrene & 2.56 & 0.008 \\
\hline
\end{tabular}

Table 1

John Wiley \& Sons 


\begin{tabular}{ccccc}
\hline & $\begin{array}{c}C \\
\left(\mathrm{~J} /\left(\mathrm{kg} \cdot{ }^{\circ} \mathrm{C}\right)\right)\end{array}$ & $\begin{array}{c}k \\
\left(\mathrm{~W} /\left(\mathrm{m}^{\circ}{ }^{\circ} \mathrm{C}\right)\right)\end{array}$ & $\begin{array}{c}\rho \\
\left(\mathrm{kg} / \mathrm{m}^{3}\right)\end{array}$ & $\begin{array}{c}\eta \\
(\mathrm{cP})\end{array}$ \\
\hline Water & 4168.9 & 0.625 & 1000 & 0.88 \\
$\begin{array}{c}\text { Culture } \\
\text { medium } \\
\text { Agar } \\
\text { gel }\end{array}$ & 4067.3 & 0.619 & 1000 & 0.94 \\
\hline
\end{tabular}

Table 2 


\begin{tabular}{ccccc}
\hline & $\begin{array}{c}\mathrm{SAR}_{\text {peak }} \text { computed } \\
(\mathrm{W} / \mathrm{kg})\end{array}$ & $\begin{array}{c}\text { Deviation with } \\
\text { respect to SAR in } \\
\text { water }\end{array}$ & $\begin{array}{c}\mathrm{SAR}_{\text {peak }} \text { measured } \\
(\mathrm{W} / \mathrm{kg})\end{array}$ & $\begin{array}{c}\text { Corrected SAR }_{\text {peak }} \\
(\mathrm{W} / \mathrm{kg})\end{array}$ \\
\hline Water & 7767 & $0 \%$ & $6484.1 \pm 380.4$ & $7780.9 \pm 456.4$ \\
$\begin{array}{c}\text { Culture medium } \\
\text { (without cells) }\end{array}$ & 7832 & $1 \%$ & $6583.2 \pm 522.6$ & $7899.8 \pm 627.1$ \\
$\begin{array}{c}\text { Culture medium } \\
\text { (with cells) }\end{array}$ & 7910 & $2 \%$ & $6686.8 \pm 701.0$ & $8024.1 \pm 841.2$ \\
Agar gel & 7725 & $0.5 \%$ & $6427.0 \pm 163.2$ & $7712.4 \pm 195.8$ \\
\hline
\end{tabular}

Table 3 


\begin{tabular}{cc}
\hline$g\left(\mathrm{~m} / \mathrm{s}^{2}\right)$ & 9.8 \\
$\beta(1 / \mathrm{K})$ & 0.003 \\
$x(\mathrm{~m})$ & $5.2 \cdot 10^{-3}$ \\
$\Delta T\left({ }^{\circ} \mathrm{C}\right)$ & 3 and 12.5 \\
$\alpha_{\text {water }}\left(\mathrm{m}^{2} / \mathrm{s}\right)$ & $1.5 \cdot 10^{-7}$ \\
$\alpha_{\text {culture medium }}\left(\mathrm{m}^{2} / \mathrm{s}\right)$ & $1.52 \cdot 10^{-7}$ \\
$v_{\text {water }}\left(\mathrm{m}^{2} / \mathrm{s}\right)$ & $0.63 \cdot 10^{-6}$ \\
$v_{\text {culture medium }}\left(\mathrm{m}^{2} / \mathrm{s}\right)$ & $0.67 \cdot 10^{-6}$ \\
\hline
\end{tabular}

Table 4

John Wiley \& Sons 


\begin{tabular}{cccccc|ccccc}
\hline$\Delta T\left({ }^{\circ} \mathrm{C}\right)$ & & \multicolumn{3}{c}{3} & \multicolumn{5}{c}{12.5} \\
$V(\mathrm{ml})$ & 0.5 & 1 & 1.5 & 2 & 4 & 0.5 & 1 & 1.5 & 2 & 4 \\
$x\left(10^{-3} \mathrm{~m}\right)$ & 1.3 & 2.6 & 3.9 & 5.2 & 10.4 & 1.3 & 2.6 & 3.9 & 5.2 & 10.4 \\
\hline$G r_{\text {water }}$ & $4.9 \cdot 10^{2}$ & $3.9 \cdot 10^{3}$ & $1.3 \cdot 10^{4}$ & $3.1 \cdot 10^{4}$ & $2.5 \cdot 10^{5}$ & $2.0 \cdot 10^{3}$ & $1.6 \cdot 10^{4}$ & $5.5 \cdot 10^{4}$ & $1.3 \cdot 10^{5}$ & $1.0 \cdot 10^{6}$ \\
$G r_{\text {culture medium }}$ & $4.3 \cdot 10^{2}$ & $3.45 \cdot 10^{3}$ & $1.2 \cdot 10^{4}$ & $2.8 \cdot 10^{4}$ & $2.2 \cdot 10^{5}$ & $1.8 \cdot 10^{3}$ & $1.4 \cdot 10^{4}$ & $4.9 \cdot 10^{4}$ & $1.15 \cdot 10^{5}$ & $9.1 \cdot 10^{5}$ \\
\hline
\end{tabular}

Table 5 